\title{
Pour une anthropologie pragmatiste et plébéienne du patrimoine : un scénario contre-hégémonique
}

For a pragmatist and plebeian anthropology of heritage, a counter-hegemonic scenario

Jean-Louis Tornatore

\section{OpenEdition}

\section{Journals}

Édition électronique

URL : https://journals.openedition.org/insituarss/449

DOI : 10.4000/insituarss.449

ISSN : 2680-4972

Éditeur

Ministère de la Culture

Référence électronique

Jean-Louis Tornatore, «Pour une anthropologie pragmatiste et plébéienne du patrimoine : un scénario contre-hégémonique », In Situ. Au regard des sciences sociales [En ligne], 1 | 2019, mis en ligne le 07 octobre 2019, consulté le 16 décembre 2022. URL : http://journals.openedition.org/insituarss/449 DOI : https://doi.org/10.4000/insituarss.449

Ce document a été généré automatiquement le 16 décembre 2022.

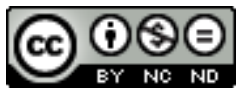

Creative Commons - Attribution - Pas d'Utilisation Commerciale - Pas de Modification 4.0 International - CC BY-NC-ND 4.0

https://creativecommons.org/licenses/by-nc-nd/4.0/ 


\title{
Pour une anthropologie pragmatiste et plébéienne du patrimoine : un scénario contre-hégémonique
}

\author{
For a pragmatist and plebeian anthropology of heritage, a counter-hegemonic \\ scenario
}

Jean-Louis Tornatore

Je remercie un des lecteurs anonymes pour ses remarques critiques et ses conseils de lectures complémentaires. Je remercie également Ellen Hertz pour ses commentaires acerbes, vengeurs et amicaux : la discussion continue!

1. Question de désignation ${ }^{1}$. Le terme " pragmatiste » est-il le qualificatif le plus approprié à l'approche du fait patrimonial que je défends? Deux raisons, à première vue, suscitent cette interrogation. D'une part, le mouvement de pensée rassemblé sous cette étiquette et qui connaît une certaine faveur en France depuis un quart de siècle est loin d'être uniforme. Même si une "association d'études pragmatistes ", Pragmata ${ }^{2}$, créée en 2014, est venue officialiser ce renouveau à partir d'une réappropriation serrée de la philosophie pragmatiste américaine, des pères fondateurs étasuniens (Charles Sanders Peirce, William James, John Dewey, Georges Herbert Mead) comme des néopragmatistes (Richard Rorty, Hilary Putnam, Richard Shusterman...), c'est la multiplicité des démarches pragmatiques ou pragmatistes qui prévaut, ou une diversité d'approches, de la sociologie de l'acteur-réseau aux tenants de l'«engagement spéculatif » qui, sans revendiquer l'appellation, partagent une proximité de posture ${ }^{3}$. D'autre part, le monde académique français, et particulièrement sociologique, a réduit jusqu'à la caricature une opposition stérile entre «critique » et "pragmatique » entendre, pour le dire vite, Bourdieu versus Boltanski dans une version sans doute datée mais qui a la vie longue (Benatouil 1999) ${ }^{4}$. Cette pluralité - dont tout pragmatiste sait s'accommoder- comme ces vieilles querelles, qui malheureusement sont institutionnellement très performatives, favorisent sans doute les perplexités et donnent prise aux charges sans nuances. Le mot a cependant le mérite de signifier des points communs entre des démarches caractérisées par une volonté de rompre avec des 
théories et des théorisations toutes puissantes qui, au lieu de faire corps avec le monde sans cesse changeant et de s'avancer en toute incertitude avec les êtres qui le composent, dans le travail de signification, n'ont de cesse de le découper, de le réduire et au final de n'en proposer que des abstractions.

2 S'agissant des études patrimoniales, un récent débat sur Academia.edu, initié par Gary Campbell et Laurajane Smith (2016) autour du texte de la communication qu'ils ont présentée lors du troisième congrès de l'Association of Critical Heritage Studies ${ }^{5}$, a semble-t-il cristallisé des dissensions nettes quant à ce que doit être une approche critique $d u$ patrimoine. Les auteurs, se réclamant du manifeste inaugural de l'association ${ }^{6}$ visant à promouvoir «un sens fort de l'engagement critique dans les questions de justice sociale et à encourager les gens à s'appuyer sur les sciences sociales en général pour étudier les musées et le patrimoine » (ibid., ma traduction) s'émeuvent de l'infestation des critical heritage studies par des théories pointées sous les appellations «post-humanism » et «new materialism» (abrégées en PH/NM) : comprendre, ainsi qu'il apparait dans la suite du texte, des courants théoriques qui trouveraient une forte inspiration dans la french theory - ici principalement identifiée à l'œuvre de Gilles Deleuze-, telles que la théorie de l'acteur-réseau (ANT), les théories nonreprésentationnelles (NRT) et seraient liés aux figures intellectuelles de Bruno Latour, du géographe Nigel Thrift, du philosophe Brian Massumi ou encore de philosophes féministes telles que Rosi Braidotti et Karen Barad. Jugés inutilement complexes, considérés comme tendant à des formulations aussi obscures que vides, comme exerçant "malheureusement" un certain pouvoir d'attraction par leur prétendue nouveauté et comme étant ainsi sujets à des références souvent purement formelles, mal digérées ou peu informées, ces travaux et courants de pensée sont visés sur un certain nombre de thèmes qui, aux yeux de leurs détracteurs, font leur unité: agentivité, particulièrement des non-humains et autres êtres du monde matériel, primauté des objets, "accent mis sur l'immanence, l'auto-organisation, les flux, les réseaux, les assemblages, concourant à l'idée d'ontologie plate » (ibid., ma traduction), intérêt pour les affects... Ces thèmes constituent pêle-mêle la cible des auteurs qui y voient le signe et le risque de l'abandon pur et simple de la posture critique, posture qui doit mettre le chercheur en capacité d'être attentifs aux seules questions qui importent, soit celles qui intéressent les gens: "Pour nous "critique" ne veut pas dire adopter quelque mode intellectuelle, mais opter pour des questions pragmatiques et clairement définies qui importent au personnes. Questions de pouvoir et d'exploitation, d'inégalité et d'idéologie, de classe, de genre et d'ethnicité, qui comptent encore pour nous » (ibid., ma traduction). Ainsi une approche critique réaliste ne doit pas surestimer le poids des objets ni des affects dans les interactions sociales mais au contraire se faire l'écho des thèmes qui prioritairement émergent des discours des personnes - «le poids écrasant de leur opinion est que ce qu'ils font est personnel, social ou culturel, et n'est pas dominé par les choses avec lesquelles ils interagissent, ou bien paralysé par les affects, bien qu'ils puissent être affectés » (ibid., ma traduction). Il n'est pas nécessaire, à ce point, de se livrer à une justification des théories et démarches incriminées quant à leur potentiel critique, tant la charge est grossière et très superficielle. Il faut savoir cependant qu'elle vise sans la nommer explicitement l'approche défendue par Rodney Harrison lequel, tout en plaidant pour une mise en perspective dialogique du patrimoine, nourrie, entre autres, des travaux de Bruno Latour et de Edouardo Viveros de Castro, la revendique comme critique - le titre de son dernier ouvrage, Heritage. Critical approaches, est suffisamment éloquent à cet égard (Harrison 2013). 
3 Si on en revient donc à la question d'entrée, on voit bien que se dessine, du moins est déclarée une césure dans le paysage académique et intellectuel du patrimoine qui interroge à la fois la posture des chercheurs, leur équipement conceptuel et leurs appuis théoriques pour rendre compte $\mathrm{du}$ fait patrimonial contemporain. La revendication d'une démarche fondée sur « les pratiques et les représentations » des humains, interagissant localement mais sous la détermination de forces de type macro telles que la société ou la culture, la classe, le genre ou la race, rejette en vrac des approches partageant le refus des dualismes puissants qui enserrent la pensée académique : sujet-objet, humain - non-humain, nature-culture. Toutefois, cette césure se faufile dans un paysage très varié de références et de positionnements analytiques, au prix d'une difficulté à pointer et à nommer précisément les oppositions. Quand bien même le mot « pragmatisme » n'est pas énoncé comme identifiant les ressources ou les postures analytiques à rejeter, il n'est pas difficile de l'y rapporter. De même qu'il est aisé de percevoir que l'approche définie comme "critique » par Rodney Harrison entretient une proximité certaine avec celle que j'appellerai donc « pragmatiste ».

2. Question de position. Bien que depuis une quinzaine d'années, dans le paysage des études patrimoniales hexagonales, quelques références au pragmatisme se remarquent, aucun courant de recherche ne s'est encore, à ma connaissance, manifesté sous cette désignation. L'anthropologie du patrimoine, au moins celle développée au sein du Laboratoire d'anthropologie et d'histoire de l'institution de la culture (LAHIC), en collaboration ou en interaction avec les institutions patrimoniales, n'a jamais témoigné d'un grand intérêt pour la pensée et la démarche pragmatistes, au mieux un intérêt poli, sans doute à première vue en raison du peu d'appétence pour la réflexion théorique et partant d'une méconnaissance des courants de pensée, des auteurs et travaux concernés. À regarder de plus près, on peut y voir un effet de la nature politique du patrimoine et des savoirs "embarqués" qui se sont développés sous l'égide de la raison patrimoniale. Si tout dispositif patrimonial - comme tout dispositif muséal - effectue un croisement entre science et administration, dans des sortes de forums « hybrides ${ }^{7}$ ", c'est au prix d'une représentation toujours purifiée des places qui y sont occupées par leurs acteurs. Les savoirs et les discours d'autorité produits en ces circonstances sont indexés sur des positions spécifiques, mutuellement reconnues et attendues comme telles: le consensus patrimonial commence ici, c'est-à-dire dans l'acceptation préalable que chacun.e parle depuis un statut qui lui confère une qualité d'expert spécifique et dans l'attente que chacun.e produise un discours conforme à ce statut. Ces forums, tel celui qu'a été feu le Conseil du patrimoine ethnologique (avec sa commission permanente) dont l'objectif officiel était d'élaborer « une politique du patrimoine ethnologique ", constituent le volet qualitatif d'une science d'État, une statistique au sens propre. En l'occurrence, universitaires et chercheurs représentants de disciplines jugées susceptibles de décrire et d'analyser les phénomènes culturels et patrimoniaux, fonctionnaires de l'administration centrale, représentants de grandes associations patrimoniales (par exemple Maisons paysannes de France) se rencontrent, échangent et débattent sur les évolutions de la société française, sur les récurrences et les singularités susceptibles d'être étudiées en profondeur et sur la définition des thèmes de recherche qui doivent permettre d'orienter et de guider l'action de l'État en matière de préservation et de valorisation des modes et des cadres de vie, des cultures et des environnements. L'identification partagée des places doit permettre que chacun, sous un objectif général, s'y retrouve dans ses propres objectifs : quand les premiers font de la science et que les troisièmes font du patrimoine, les deuxièmes font et 
servent la politique du patrimoine. Avec la notion d'objet-frontière, Susan Leigh Star (Leigh Star \& Griesemer 1989 ; Leigh Star 2010 ; Trompette \& Vinck 2009) a fort bien montré qu'il est possible de collaborer à la réalisation d'un projet tout en poursuivant ses propres objectifs, et sans qu'il soit nécessaire d'y adhérer ou d'endosser la chasuble du croyant $^{8}$, chacun se liant par intérêt spécifique dans le temps et l'espace de ce projet. Certes, la rencontre est fragile et chacun est toujours susceptible de remettre en question sa place et sa participation : la crainte pour les uns de se faire politiquement manipuler, de perdre la capacité de produire un savoir "objectif», de tomber dans l'idéologie reste toujours présente, quand les autres peuvent s'exaspérer de voir l'action patrimoniale compromise par un excès de réflexion ou d'autres estimer qu'il n'est pas du ressort d'un ministère de financer de la recherche qui risquerait d'être « désintéressée », mais c'est justement à l'espace du forum hybride qu'est dévolu un efficace à assurer, conjointement, à chacun l'intégrité de sa position dans la place qui lui est attribuée et à l'institution commanditaire la satisfaction de son objectif de politique publique.

5 Le savoir produit est essentiellement documentaire : il décrit et objective les propriétés des objets éligibles à la distinction patrimoniale. Ainsi, il contribue à l'instauration de l'être patrimonial en tant que maillon ou étape inaugurale de ce que la doctrine patrimoniale définit comme une chaîne liant connaissance-conservation-valorisation. À chacune de ces trois étapes sont censés correspondre ses spécialistes - pour autant, un conservateur peut tout à fait émarger aux trois -, ceci pour signifier qu'elles - et leurs représentants - doivent être solidaires. La science est intéressée à la chose patrimoniale pour ses capacités d'objectivation et parce qu'elle s'élabore comme neutre et désintéressée. Toutefois, lorsque se rompt l'équilibre solidarité-désintéressement, quand l'argument d'une science désintéressée se fait par trop insistant et dénoue en somme la solidarité nécessaire des acteurs, l'institution commanditaire en vient à défaire le forum. C'est bien ce qui s'est produit pour la Mission du patrimoine ethnologique (MPE), disparue en 2010 de l'organigramme du ministère de la Culture au terme de trente ans d'existence et d'une décennie de lent désengagement. Logiquement, la fin de cette aventure à la fois scientifique et institutionnelle a suscité et suscite encore un retour réflexif ${ }^{9}$, sinon un désir d'histoire. Mais quelle histoire promouvoir? Pour répondre à cette question, il faut considérer la singularité bifide du dispositif: la partition entre l'action dite "en centrale " (soit à Paris, au sein du ministère et occupée principalement à l'organisation du forum hybride) et l'action en région. Dite et convenue, cette partition n'a cependant jamais été trop creusée, voire a été atténuée tant elle pouvait être sulfureuse et mettre en danger le statu quo du forum : c'est bien depuis ou dans l'action en région qu'a pris naissance un positionnement pragmatiste ; l'action en région a été, pour certain de ses agents, lesdits « conseillers à l'ethnologie", propice à l'exploration d'une posture de recherche de sensibilité pragmatiste : penser le patrimoine et l'action patrimoniale à partir de cette position singulière - pour le dire classiquement, une position de recherche-action. On ne s'étonnera guère que la proposition d'une "histoire positiviste ${ }^{10}$ " soit énoncée du point de vue de l'action en centrale : il ne s'agit ni plus ni moins que de la réitération de la posture forte du partage des attentes, professant la purification des discours tenus dans l'enceinte du forum et la poursuite du geste pastoral (Barbe 2013) qu'a été la MPE. La désignation " positiviste » a cependant de quoi surprendre tant le terme est associé à une période datée de l'historiographie française (soit la seconde moitié du XIX et le début du $\mathrm{XX}^{\mathrm{e}}$ siècle), même indûment, comme l'a très bien montré Charles-Olivier 
Carbonell (1978), et attribué "aux historiens chartistes et sorbonnards du temps" (ibid. : 173) ${ }^{11}$. Pour autant, positiviste ou plus exactement "positive» (ibid. : 182 sq.) $)^{12}$, c'est-à-dire professant l'amour du fait, l'exploitation exhaustive des documents, qu'ils soient écrits ou obtenus par entretiens, et la méthode prosopographique, cette histoire s'inscrit dans une tradition monographique qui doit concourir à éclairer un pan de l'histoire des institutions: une tradition en somme d'histoire institutionnelle et par conséquent étatique. Il ne s'agit pas tant pour moi de disqualifier ce projet que de souligner la «logique " dont il peut se prévaloir; il s'agit de le situer, c'est-à-dire d'éclairer les relations qui le motivent ou qui le justifient.

Une histoire positiviste ou positive de la Mission du patrimoine ethnologique ne saurait être considérée en soi et ne saurait avoir une autonomie ou une existence détachée (objective) ; elle est indissociablement liée au mode de fonctionnement qui a prévalu et qui a alimenté une doxa institutionnelle qui en retour la légitime. Finalement, tout se passe comme si le projet de cette histoire poursuivait l'ignorance ou la mésestimation d'une réflexion qui, tentant de penser le patrimoine et les actions patrimoniales (Barbe 2003) depuis l'action en région, s'interrogeait sur ce qu'elle - c'est-à-dire la position singulière qu'elle signifiait - faisait faire. Tout se passe comme si le constat fait par un ethnologue renommé, ayant présidé pendant plusieurs années les travaux du Conseil du patrimoine ethnologique, "nous autres ethnologues, sommes embarqués " (Fabre 1994 : 148), avait été oublié sitôt énoncé : il y a loin entre voir l'action patrimoniale depuis le rivage ou quelque position terrestre ou sommitale, et la vivre en haute mer, au creux et à la crête des vagues. S'il y a une histoire à écrire, c'est celle qui ne craindra pas de plonger dans le concret et le présent de cette articulation - qui en son temps fut nouvelle - entre action patrimoniale et action en région et qui donc se confrontera aux manières singulières dont a été négociée la double identification d'acteur et de chercheur. Sur cette hybridité s'est notablement cristallisée l'accueil fait aux rares analyses produites : s'il était clair, avec une constance remarquable, pour un «chef » de la Mission du patrimoine ethnologique ou un quelconque représentant de l'administration centrale qu'un conseiller à l'ethnologie n'était qu'un agent du patrimoine, au mieux administrateur de la recherche, quelques-uns - rares cependant parmi les conseillers à l'ethnologie ont estimé n'avoir jamais cessé d'être chercheur tout en étant agent, et avoir considéré que leur statut singulier nécessitait un surcroît de réflexion, que leur pratique appelait la recherche de nouveaux postulats théoriques et de nouveaux cadres conceptuels. S'il y a donc une histoire à écrire, elle gagnerait grandement à être pragmatiste, c'est-à-dire à restituer au moins les tâtonnements de cette recherche, non pas théoriquement mais dans le fil des situations indéterminées ou des expérimentations dans lesquelles s'engage(ait) l'agent-chercheur..$^{13}$ Elle s'attacherait à contribuer à une sociohistoire de l'action publique, en suivant ses acteurs, que ce soit en restituant le quotidien du conseiller à l'ethnologie - une histoire au ras du sol administratif, ethnologique et culturel - ou bien en (re)parcourant le processus de construction du patrimoine ethnologique comme problème public au sein des instances centrales ${ }^{14}$.

3. Question d'expérience. Lorsque j'envisage la rencontre des ethnologues avec le patrimoine, c'est à partir de cette expérience singulière de confrontation entre une action en région indexée sur un statut administratif et répondant à une politique d'État, et des actions à visée patrimoniale ou visant l'attribution d'une valeur patrimoniale. Une rencontre donc « en pratique » - et non pas, comme cela peut l'être dans un cadre académique, à partir d'une «simple» position de recherche («faire 
l'anthropologie d'une situation patrimoniale »). En d'autres termes, une confrontation, pour l'agent-chercheur, à l'objectivation de la culture - c'était l'argument de naissance du Lahic - ou du passé, ou, mieux, à leur politisation depuis une position politique c'était l'argument que Noël Barbe et moi avons d'emblée proposé. Ce que cette rencontre a fait faire se lit dans une forme de déplacement par rapport à une doxa patrimoniale: entreprendre une approche du patrimoine qui soit tout à la fois inclusive, située et engagée; et qui peut être dite pragmatiste en tant que le pragmatisme est une pensée des relations et des conséquences fondée sur un principe d'indétermination des situations sociales - un principe de «faillibilisme » selon Albert Ogien $(2014: 565)$.

8 Premièrement, le caractère inclusif, ou bien ouvert, renvoie au souci de ne pas répondre au découpage a priori de la réalité patrimoniale, signifié par la distinction de catégories de patrimoine (monumental, ethnologique, naturel, etc.) comme à la pluralité de termes disponibles pour dire une forme de relation au passé (patrimoine, mémoire, histoire, territoire), à la culture ou à ce qui nous environne $\mathrm{e}^{15}$. On doit se demander ce que signifient ou deviennent «le passé», "la culture», «l'environnement » ou « la nature » dès lors qu'on les saisit sous une injonction ou une qualification qui aujourd'hui est dite patrimoniale. Nonobstant la difficulté à dégager le mot patrimoine de sa structure de signification, il importe de spécifier ce que veut dire instaurer un être comme patrimoine : plus exactement, en renversant la question, de quel désir récurrent, par-delà les objets, les dispositifs et leur historicité, le patrimoine est-il le nom ${ }^{16}$ ? On a avancé la valeur de pérennité qui questionne la durabilité des choses et par laquelle, semble-t-il, nos sociétés engagent leur rapport au temps (Fabre 2014). Nous plaçant du côté des actions engagées par des personnes et/ou des collectifs, nous lui avons préféré l'idée d'attachement, c'est-à-dire moins une valeur qu'un geste signifiant une attention à ce qui est considéré par les personnes ou les collectifs comme ayant du prix, comme étant ce qui compte, ce qu'il leur importe de sauvegarder et ce à quoi elles/ils se lient dans une relation d'instauration ${ }^{17}$ : en d'autres termes, l'attachement dit que «ce à quoi nous tenons nous tient ${ }^{18}$ »; il est un geste d'intensification de l'importance (Debaise 2015) qui permet de souligner l'agentivité des choses dans la relation instaurée avec elles.

Deuxièmement, l'approche est située et, en ce sens, indexée sur les formes singulières, provisoires et révisables de la relation instaurée. Si le patrimoine se concrétise dans des attachements dans le présent des collectifs et se réalise dans la "pédagogie » de ses objets - i.e. ce qu'ils font faire-, alors son actualité - l'actualité de la relation d'attachement - importe. L'un des apports de la démarche pragmatiste a été de souligner que l'opérativité du patrimoine se joue dans sa valeur de présence - actualité et actualisation-, non dans sa valeur de transmission: transmettre n'étant qu'une modalité de la «fin-en-vue » (Dewey 2011) de l'action patrimoniale, non une «fin-ensoi » qui déterminerait cette action. En ce sens, la notion deweyenne de valuation (ibid.), refusant de trancher a priori entre le verbe et le nom, est transposable au patrimoine, qui ainsi apparaît manifestement comme une activité située. S'engager dans une action qui vise à attribuer à un objet ou à un être une valeur patrimoniale, ou à confirmer cette valeur, est un processus incertain par lequel les individus à la fois font valoir et découvrent ce à quoi ils tiennent. Pas plus que n'importe quelle autre valeur, la valeur patrimoine n'est un donné, ni pour ceux-ci qui se découvrent héritiers, ni pour l'anthropologue qui n'aurait qu'à mettre cette valeur au jour de façon empirique (ethnographique) ; elle n'est jamais définitivement acquise, elle n'est pas inscrite dans 
l'objet ou l'être au point de le protéger définitivement - si elle fait faire, elle ne fait pas être -, elle est toujours à justifier - ce à quoi s'emploient, entre autres, les institutions patrimoniales ${ }^{19}$. Bref, le patrimoine ne se transmet pas, il s'actualise, il ne se transmet qu'à condition de s'actualiser. L'histoire du patrimoine pourrait ainsi être résumée à un incessant travail de preuve, de justification. Elle est émaillée d'inscriptions et de désinscription, de restaurations et de dérestauration, et possède la propriété de "ramasser le temps", de le condenser ou de le plier, lorsque des acteurs de la patrimonialisation font littéralement ou concrètement intervenir (ils les invoquent et les convoquent) des acteurs du passé comme du futur ${ }^{20}$. Le motif du présentisme (Hartog 2003) ne serait pas sans intérêt de ce point de vue, s'il n'était singularisé en tant que régime d'historicité succédant à d'autre régimes d'historicité et caractérisant notre époque et s'il ne procédait de l'évaluation négative d'un présent dilaté et monstrueux. Le pragmatisme permet de dire que l'activité patrimoniale ne cesse pas d'être présentiste - pour peu que l'on fasse du présent le lieu plein de toutes les expériences ${ }^{21}$.

Une approche pragmatiste du patrimoine consiste à s'interroger sur le genre d'expérience collective qui se noue sous cette désignation, et en quoi consiste la présence qu'elle instaure. Qu'est-ce que l'expérience patrimoniale? Qu'est-ce que faire une expérience de patrimoine? Qu'est-ce que le patrimoine compris comme une expérience, au sens de John Dewey (2012b), c'est-à-dire comme une forme singulière de la relation entre les humains et le monde. Considérer le patrimoine comme expérience, c'est faire bénéficier au patrimoine la qualité « à double détente » (James $2005: 40)$ du mot « expérience ", en tant qu'il dit à la fois le contenu et la pensée, le quoi - ce dont les humains font l'expérience - et le comment - « les processus qui sont impliqués dans les expériences qu'il font» (Dewey 2012b: 39) ${ }^{22}$. Le patrimoine est tel qu'il est " expériencé » - dont il est fait une expérience -, il est à la fois la chose " expériencée " et les relations signifiées et organisées dans et par l'expérience. Aussi est-il nécessaire de prendre en compte les prépositions qui engagent (et commentent) l'expérience patrimoniale : ce sont, note Bruno Latour (2012: 69), des «clés d'interprétation » du type de relation instaurée dans la transaction avec le monde : le patrimoine, ce n'est jamais un patrimoine-tout-court, ce n'est toujours que du patrimoine-de, du patrimoine-pour, du patrimoine-avec, du patrimoine-contre, etc. Le patrimoine n'étant pas limitable à son contenu, on ne saurait non plus le cantonner à une expérience du passé (Voisenat 2016) ou même à une expérience de la culture, sous couvert d'objectivation ou même de politisation - ce serait rater la singularité de la relation instaurée. Aussi, il importe d'aller plus loin, de voir avec et au-delà des « objets » et de comprendre le sens et l'efficace de l'attachement instauré dans et par l'expérience patrimoniale proprement dite, de situer pour cela cet attachement dans un enchaînement d'expériences qui engagent les activités sociale, mentale et émotionnelle, et se déploient dans une diversité de registres: esthétique, artistique, éthique, politique, marchand. Considérer le patrimoine en son actualité, en somme dans ses différents modes d'existence, constitue un geste premier permettant d'interroger son opérativité et sa puissance d'agir (son agentivité). 
Le Poulain de Pézenas, juillet 2017.

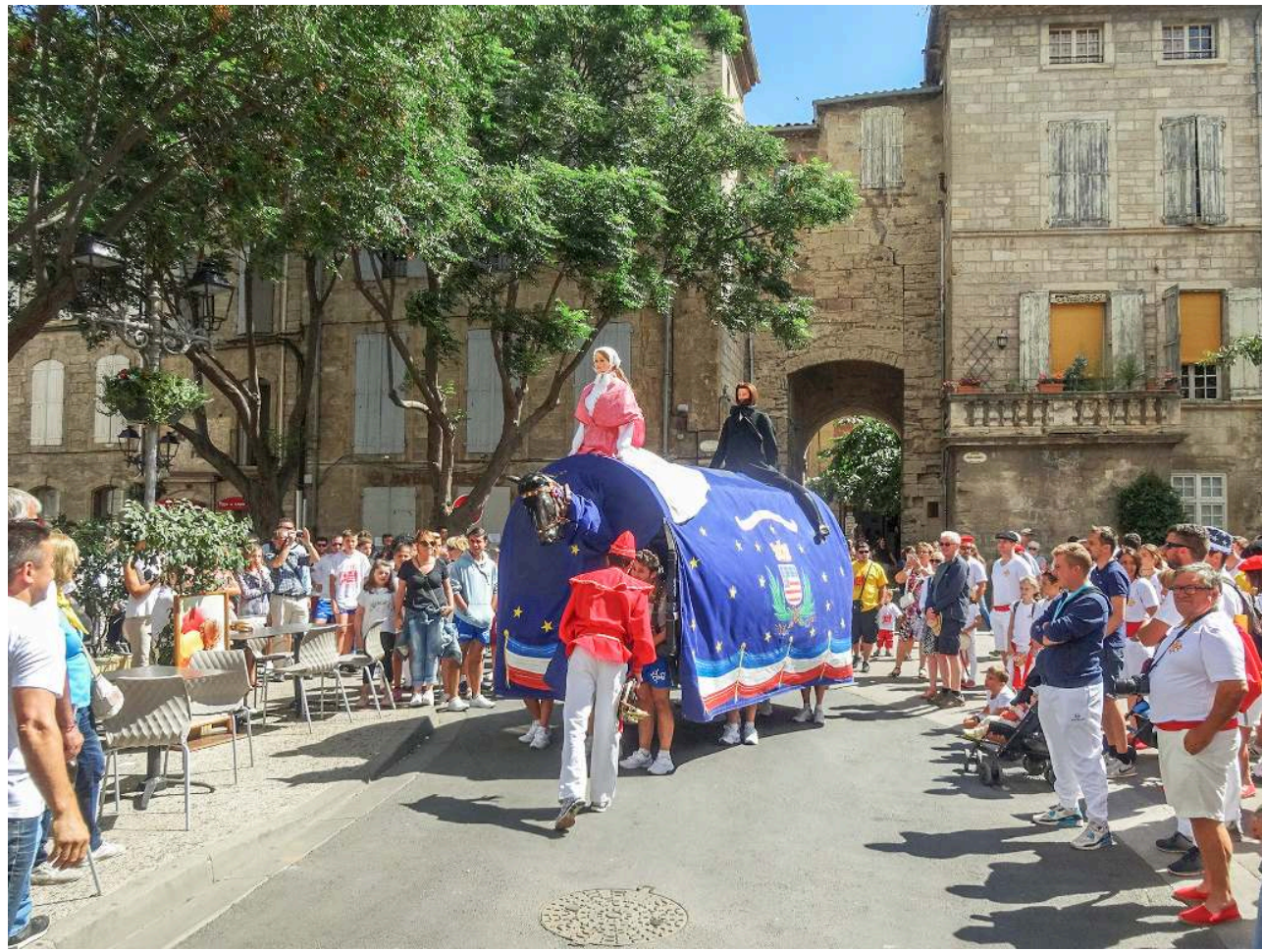

Faire l'expérience du patrimoine : le Poulain de Pézenas est un animal « totémique » inscrit sur la Liste représentative du patrimoine culturel immatériel en tant que partie de l'élément « Géants et dragons processionnels de Belgique et de France » (Unesco 2005 et 2008). Être carnavalesque, il sort ici en juillet 2017, à l'occasion d'une « Ronde des géants et des totems », manifestation festive qui accompagne la création de Totemic, fédération des totems catalans et occitans. Lié à une ville ou un village, le totem est considéré comme le « boute-en-train de la fête » : dans la perspective de la fédération, il est conçu comme le représentant de la civilisation d'òc via ses deux valeurs : la convivialité et le partage (http://totemic.occitanica.eu/fr/totems/).

(c) Jean-Louis Tornatore.

4. Question d'engagement. Le caractère situé de l'approche pragmatiste s'applique également à l'enquête scientifique : d'être située, la connaissance produite se réalise à l'opposé de celle élaborée sous l'injonction d'une objectivité neutre ou d'une neutralité objective $^{23}$. On touche ici au troisième trait qui concerne l'engagement, une question qui a nourri de manière centrale la réflexion menée depuis l'action en région. La notion d'implication a connu un certain succès dans notre petit milieu du patrimoine ethnologique, sans pour autant faire l'objet de développement substantiels. Faire une " ethnologie impliquée ${ }^{24}$ » - et non appliquée -, c'était reconnaître la présence active de l'ethnologue, ou du spécialiste en ethnologie, active au sens où il ne pas se contente pas de documenter des êtres éligibles à la distinction patrimoniale mais est invité, par ses fonctions, à participer à l'engagement d'actions patrimoniales, à participer de l'expérience patrimoniale. Engager une action patrimoniale, c'est s'y engager. Le thème de la participation ou de l'engagement participatif a été formulé une première fois dans l'idée que ce que j'ai appelé un « tournant réflexif » avait affecté (grosso modo au passage des années 1980-1990) l'institution du patrimoine ethnologique (Tornatore 2004b). Ce thème a connu par la suite une plus grande publicisation avec la proposition d'un «tournant participatif » qui affecterait de nos jours le patrimoine dans le cadre d'un " effet $\mathrm{PCI}^{25}$ ». Mettons que le PCI a explicitement mis en avant un thème que nous avions largement souligné - mais au prix de farouches oppositions dont on pourrait 
dire, en langage syndical, qu'elles relevaient de la défense d'intérêts sectoriels -, celui du partage des expertises. Ce qui est concevable aujourd'hui était inaudible hier, ce qui est concevable aujourd'hui l'est au prix de l'oubli d'hier. La raison académique n'a pas manqué de minorer cet apport en concédant sagement le développement d'une ethnologie du patrimoine ${ }^{26}$, laissant le débat se caricaturer dans une tension entre la posture de l'ethnologue critique (celui qui dévoile et dénonce) et celle de l'ethnologue embedded qui rédige des fiches d'inventaire au détriment d'une réflexion avancée sur ce que s'engager veut dire et sur les conditions de possibilité d'une «anthropologie patrimoniale » ou plus largement d'une science patrimoniale - même si l'expression n'est pas dénuée d'ambiguïté.

Il faut ici tout de même entrer de plain-pied dans la question politique. Avant tout, on remarquera que se développent aujourd'hui une nouvelle histoire et une nouvelle géographie qui se donnent l'environnement pour objet et qui se disent respectivement "environnementales», et non «de l'environnement» (Fressoz, Graber, Locher \& Quenet 2014; Chartier \& Rodary 2015a) comme manière d'engager une démarche réflexive, d'historiciser la relation à l'objet et de reconnaitre que cet objet " travaille » son savoir et transforme la discipline scientifique qui le produit. Dans leur introduction au Manifeste pour une géographie environnementale, Denis Chartier et Estienne Rodary (2015b) développent à propos de la géographie confrontée à l'environnement une argumentation dont les termes rappellent singulièrement la confrontation de l'ethnologie au patrimoine - science impliquée, faussement apolitique, se réfugiant dans le viatique de la distanciation objectivante -, au point qu'une simple conversion terminologique suffit à dresser le portrait de l'une comme de l'autre discipline : « La réticence des géographes français à aborder politiquement la question écologique " (ibid. : 27) peut aisément être transformé en «La réticence des ethnologues français à aborder politiquement la question patrimoniale ». La géographie environnementale qu'ils appellent de leurs vœux, "une géographie environnementale réellement politique " (ibid.: 29), "cosmopolitique, post-déterministe, globale mais rugueuse, située, juste, sensible, relâchée " (ibid.: 46), ne se réclame pas explicitement du pragmatisme. Elle est cependant très proche de la science patrimoniale postulée ici, en tant qu'elle est étroitement connectée aux thèmes et aux positionnements théoriques qui dessinent aujourd'hui les linéaments d'une autre manière de faire science, « devant » les enjeux de notre époque, c'est-à-dire confrontée à eux. Les liens entre environnement et patrimoine d'ailleurs ne manquent pas, quand le premier est susceptible de faire l'objet d'une politique patrimoniale et que le second peut être une modalité du premier, peut être traité comme "ce qui nous environne », et quand l'un et l'autre sont considérés comme rapports sociaux et politiques.

La perspective d'une anthropologie patrimoniale "réellement politique ", c'est-à-dire d'une anthropologie transformée par le patrimoine, peut être développée en partant de la question de l'autorité. Ce que nous a appris l'implication participative dans le cadre d'expériences patrimoniales, c'est la nécessité de reconsidérer la position d'autorité du chercheur. Envisager un forum hybride en tant que lieu de partage des expertises, ce peut être aussi dans le sens d'une mise en commun en sorte que les expertises ou les autorités (le fait d'être l'auteur de quelque chose et de s'en prévaloir) s'additionnent au lieu de se neutraliser - lorsqu'elles se neutralisent, c'est en général au bénéfice des plus fortes. Pour autant, la question n'est pas tant de trouver la bonne posture, celle qui conduit à ne pas dénigrer ou disqualifier les savoirs non scientifiques, la connaissance 
pratique ou sensible, à accepter que ces savoirs viennent littéralement s'interposer ${ }^{27}$, que d'échapper à de tels partages des savoirs en produisant une nouvelle connaissance fondée sur de nouvelles épistémologies. La position de revendication de l'autorité scientifique, celle qui règle et hiérarchise les places, sert les postures globalement objectivistes et nourrit la tentation d'une histoire positiviste : la mise en coupe réglée de la réalité, l'imposition de savoirs surplombants, la dénonciation au nom de la raison de toute forme de relation empathique, la soumission plus ou moins tacite au dogme de la neutralité axiologique... vont de pair avec l'administration de populations et de territoires. Dès lors la question de l'autorité doit être nécessairement connectée au projet politique inhérent à l'activité patrimoniale. La position d'«ethnologue embarqué " n'a pas manqué d'être dénoncée comme relevant de la "science de gouvernement » (Laferté \& Renahy 2003 : 239) : l'assertion pouvait être vraie du point de vue de l'activité du dispositif central de la Mission du patrimoine ethnologique, elle était douteuse sinon fausse du point de vue de l'action en région. Elle avait cependant le mérite de dire que la participation à une opération de politique publique ne pouvait pas ne pas être politique. Tout comme le soulignent Denis Chartier et Estienne Rodary (2015b : 26) à propos de la géographie, le problème est que ce lien au politique a toujours été vécu et formulé "selon des considérations qui se disaient apolitiques » : on connaît la musique anthropologique, son grand art du document comme épreuve esthétisée et par conséquent réussie de la réalité, son art du conte, aussi, qui, poussé à son sommet, n'a pas son pareil pour endormir debout un auditoire acquis, fasciné et conquis. L'ethnologie, comme n'importe quelle autre discipline - l'histoire de l'art par exemple -, n'a jamais été neutre, a fortiori lorsqu'elle se déclarait telle: elle a pu être engagée dans une politique publique précisément en raison de cette capacité à présenter ses agents purs de toute influence, de toute détermination extérieure, et à livrer clés en main une description fidèle de la réalité culturelle. Posons alors le problème à partir de la question politique : quelle politique, quel sens politique émerge d'une réflexion sur l'autorité scientifique, sur l'acceptation par le chercheur de la remettre en jeu, sur la recherche d'une justice épistémologique (voir plus bas) et sur la conviction assumée que la science est de la politique continuée par d'autre moyens? Notre approche pragmatiste entend se couper des soubassements politiques de l'ethnologie du patrimoine liés à l'État et à ses missions d'administration des populations, pour proposer d'en confectionner d'autres.

14 5. Question de problèmes publics. L'histoire du patrimoine en Occident, et plus particulièrement de l'émergence d'une sensibilité patrimoniale au XIX ${ }^{e}$ siècle, est immanquablement envisagée, au moins dans la tradition française, du point de vue de la collectivité nationale et de l'État-nation, ce qui empêche sans doute d'écrire explicitement l'histoire du patrimoine comme une histoire politique, c'est-à-dire comme une histoire d'arraisonnement. Bien que depuis une vingtaine d'années, de nombreux travaux aient souligné « la vivacité des initiatives provinciales », et le rôle en cela des folkloristes, des érudits locaux, des académies de province et des sociétés savantes (Fureix \& Jarrige 2015 : 126), domine grosso modo le schéma d'une conception nationale-patrimoniale ${ }^{28}$ - concourant à la célébration de la patrie-patrimoine (Poulot 2001) - progressivement battue en brèche, au cours du XXe siècle, par la profusion des initiatives de conservation ou de sauvegarde, et par la diversification de la palette patrimoniale. Globalement acceptable, ce panorama historique mériterait néanmoins d'être revu dans une perspective moins téléologique, à la lumière d'une modernité saisie au prisme de la «discordance des temps ${ }^{29}$ ». La relation entre patrimoine et 
capitalisme n'a jusqu'ici guère retenu l'attention, à l'exception de Marc Guillaume (1980) qui a vu dans le patrimoine un moyen de compenser le rapport utilitaire à l'objet promu par le capitalisme industriel. De manière ambiguë sans doute, l'État a offert un dispositif de réparation en instaurant une forme d'appropriation commune (ou en commun) dessinant les contours d'une puissante entité symbolique de reconnaissance et d'appartenance, la nation, mais sans remettre en question les règles de la propriété privée qui régissent l'ordre économique ${ }^{30}$, et en se faisant apôtre de la religion du progrès. Pas de patrimoine sans capitalisme : en ce sens, l'État contribue à faire du monument puis du patrimoine un problème public ${ }^{31}$, dont il a l'entière gestion et qui est en grande partie entre les mains des élites sociales et culturelles. La saisie en termes de "situation problématique ", d' " arène publique ", de "problème public " et de «champ d'expérience» (Cefaï 2016; Cefaï \& Terzi 2012) ${ }^{32}$ permet de comprendre la mobilisation d'intellectuels et d'écrivains (Chateaubriand, Victor Hugo) pour la cause des monuments et leur instauration (Guizot) dans un culte moderne, national et laïc. Elle ouvre également la perspective d'un chantier de recherche quant à des formes concurrentes ou alternatives, à des manières différentes de faire valoir des attachements - ce, dans l'éventualité où le régime d'historicité de la modernité n'irrigue pas de la même manière les multiples composantes de la population nationale, à supposer qu'il le fasse. Des documents très riches telle la correspondance de Prosper Mérimée (1998) avec Ludovic Vitet montrent que l'espace public du monument à la construction duquel l'écrivain polygraphe participe doit composer avec des sensibilités situées qui ne sont pas sans évoquer les émotions patrimoniales d'aujourd'hui ${ }^{33}$. Plus largement, il convient pourtant de ne pas en rester au schéma classique "âge du monument-âge du patrimoine» (Fabre 2013), qui reste indexé sur la saisie institutionnelle (et académique) de la relation au passé, mais d'ouvrir la focale afin de mieux comprendre les attachements liés au temps, c'est-à-dire aux manières d'habiter le temps - ou, si l'on veut, aux régimes d'historicités - : relier dans ce questionnement le rapport industriel au monde naturel, les nouvelles perceptions du paysage - une sensibilité au spectacle de la nature -, les ruptures esthétiques avec les canons et le système académique dominants - une sensibilité perceptive plus qu'imaginative -, la tension entre cette modernité esthétique et la modernité de la culture « industrielle »de masse : «marchande, sérialisée, adossée à un large public alphabétisé » (Fureix \& Jarrige 2015 : 171). 
Patrimoine et capitalisme 1 : Concorde au musée de l'Air et de l'Espace.

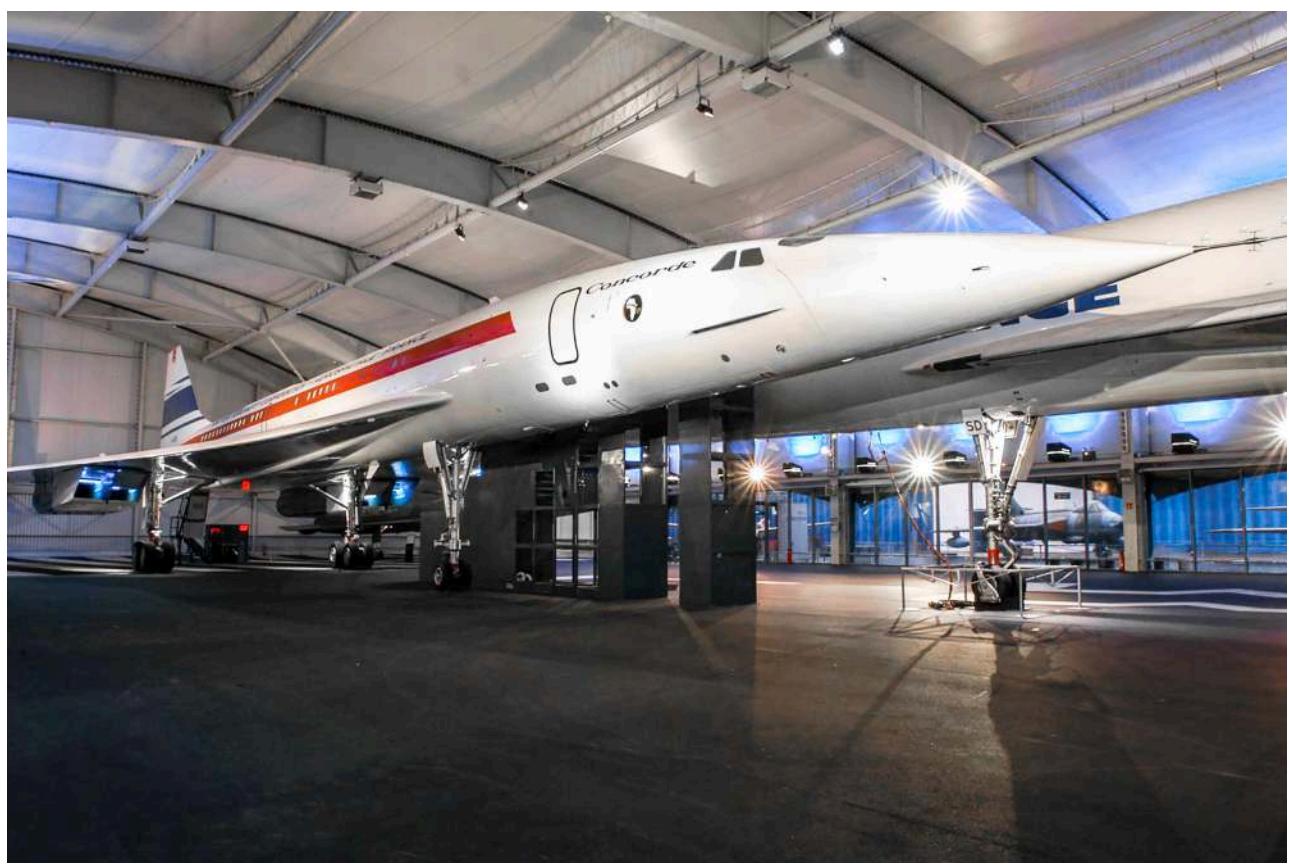

Selon Marc Guillaume, la conservation - portant la valeur cardinale de pérennité - viserait à compenser la destruction qui accompagne le développement industriel, tout en célébrant la société de consommation et de la croissance triomphante. Concorde, entré au musée en 1973 en tant que chefd'œuvre de technologie, serait ainsi l'expression parfaite d'une nouvelle sensibilité patrimoniale pour les objets de la culture industrielle.

(c) Alexandre Fernandes / cliché musée de l'Air et de l'Espace - Le Bourget.

15 C'est ainsi qu'il reste à écrire une histoire du patrimoine en tant que problème posé devant les évolutions techniques et économiques qui contribuent à la transformation de la France du XIX ${ }^{e}$ siècle, une histoire qui, ainsi, s'inscrirait dans le travail de revisite de ce siècle, et de mise en cause d'une vision par trop linéaire et reposant sur le schéma indiscuté d'une société tout entière portée par le dogme du progrès (ibid.). L'histoire environnementale comme la nouvelle histoire des techniques (Jarrige 2014) s'attachent en particulier à montrer les dissonances dans ce panorama trop lisse, en distinguant l'éveil, très tôt, d'une conscience environnementale et d'une critique du progrès technique. Comme l'écrivent Emmanuel Fureix et François Jarrige (2015: 48), «à l'ancien récit progressiste, qui voyait le XIX ${ }^{\mathrm{e}}$ siècle comme celui de la conquête de la liberté, de la démocratie, de l'émancipation à l'égard des pesanteurs de la nature, a succédé une lecture tragique où la démesure, l'exacerbation de la puissance et la prédation sont les sources de nouvelles inégalités et de catastrophes incessantes ». Et de poursuivre en note : «Ce nouvel imaginaire politique et écologiste accompagne une nouvelle histoire environnementale dynamique qui réinvente l'écriture du passé à l'aune des préoccupations du présent » (ibid.). 


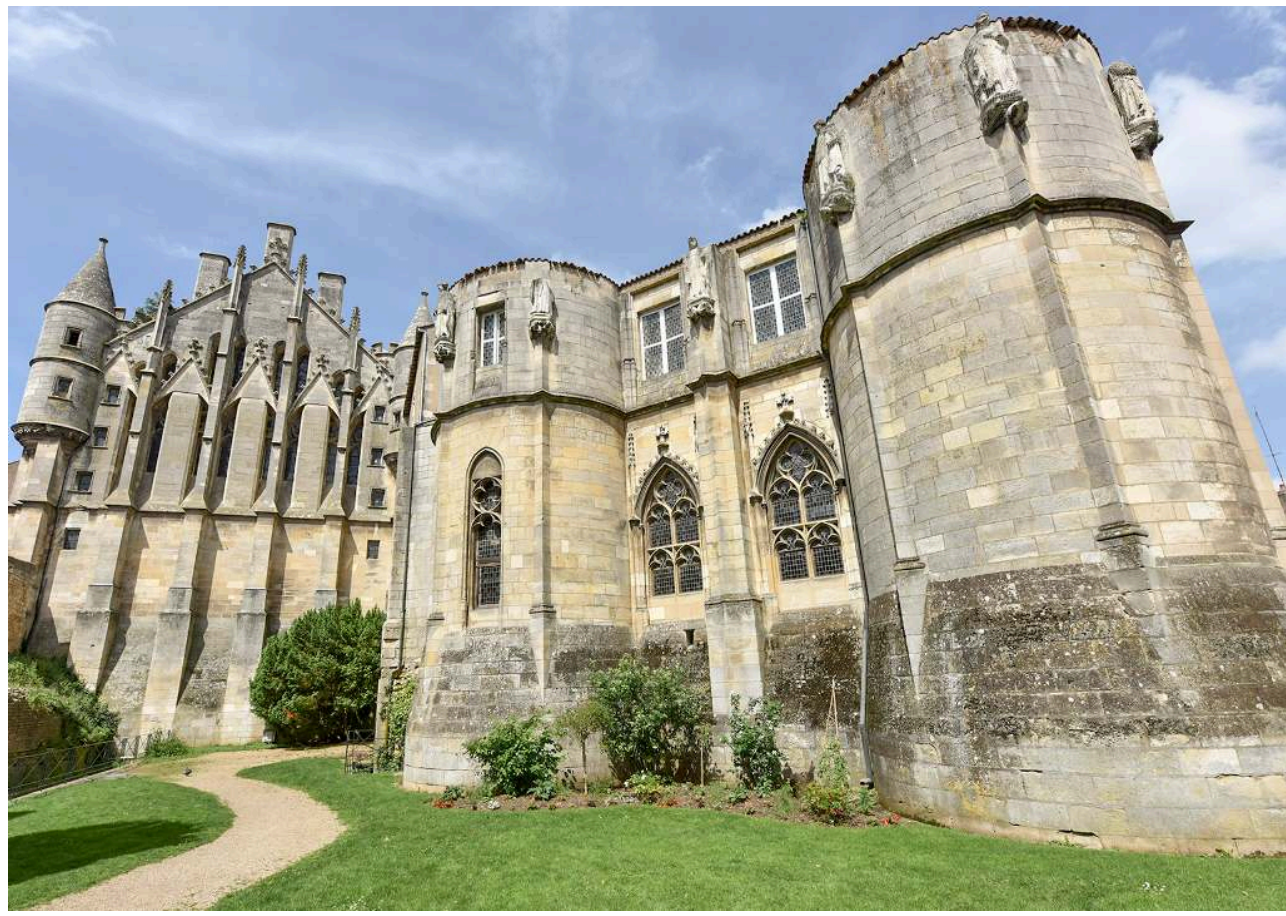

" J'ai vu aujourd'hui la tour Maubergeon et la façade du Palais de Justice qu'on veut isoler. Cela me paraît fait vers 1400 ; c'est un grand et gros édifice, un peu lourd, assez noble ; au demeurant intéressant. Le sommet des cours est couronné de grande statues sculptées à l'effet qui représentent dit-on des vicomtes de Poitiers. La plupart sont fort maltraitées. Quelques-uns ont perdu leur tête [...]. Il y a dans ce moment un enthousiasme extraordinaire à Poitiers pour la conservation du Palais de Justice. L'opinion publique s'est prononcée si vivement, m'a dit le préfet, que le Conseil municipal a hautement annoncé l'intention de contribuer par une forte allocation à l'isolement de cet admirable monument que les Poitevins viennent de découvrir à ce qu'ils croient » (Prosper Mérimée, lettre à Ludovic Vitet, 12 août 1845, in Mérimée 1998 : 148-149).

(c) Ville de Poitiers.

Sans doute le patrimoine est-il par définition anachronique, en tant qu'instaurant le passé à l'aune du présent. On a cependant vu que notre approche est moins focalisée sur la seule question du passé que sur celle du temps, en ses différentes conceptions, et sur la manière dont s'y jouent les attachements à des êtres plus ou moins fragiles. D'hier à aujourd'hui, le patrimoine est l'indice d'un trouble, d'une inquiétude, d'un « concernement » au regard de la fuite du temps, en sorte qu'il conduit des personnes, un public, à le construire en situation problématique pour la résolution de laquelle elles se mobilisent : définir le problème, en déterminer les causes, en repérer les facteurs, en établir les responsabilités... telles sont les modalités d'une dynamique collective d'enquête "qui fait à la fois émerger un problème et son public»(Cefaï 2016: 27) ${ }^{34}$. Certes, les préoccupations d'hier ne sont pas celles d'aujourd'hui, mais celles d'aujourd'hui invitent à appréhender celles d'hier et à les y comparer ; en même temps, elles invitent à s'interroger sur la capacité des catégories à franchir les temps et à épouser les préoccupations présentes. Interroger l'institution du patrimoine à l'aune des préoccupations du présent, c'est évaluer hier comme aujourd'hui son aptitude à y répondre, en d'autres termes à exprimer les problèmes publics de son, de notre temps. Alors qu'est évidente la profusion des catégories, des acteurs et des situations de patrimoine, alors que le parti a été pris d'une approche inclusive, et que le thème du vivant (préserver la culture comme patrimoine vivant, préserver le vivant comme 
patrimoine de l'humanité) est désormais un enjeu central des actions patrimoniales, il est devenu pour nous nécessaire d'évaluer l'opérativité du patrimoine devant les grandes questions politiques et environnementales de notre siècle. Cette évaluation s'appuie sur quatre prémisses :

1. Les institutions étatiques ou publiques du patrimoine ne sont plus au cœur de l'activité patrimoniale et ne sont plus un lieu d'où penser le patrimoine. Ce point est inhérent au fait que l'État a changé de forme et de fonction: d'agent régulateur du capitalisme, il est devenu, en se faisant l'apôtre de la compétition généralisée, l'agent de la dure loi de la mondialisation (Dardot \& Laval $2014: 14)^{35}$.

18 2. La mainmise capitaliste sur le patrimoine n'a jamais été aussi forte, quand la relation entre patrimoine et capitalisme ne relèvent pas ou plus de la simple logique compensatoire : le patrimoine suscite la constitution de rentes de monopole (Harvey 2008), participe pleinement d'une économie de l'enrichissement (Boltanski \& Esquerre 2017) et contribue ainsi au plein développement des industries créatives, du luxe et du tourisme. Cela n'a sans doute pas été assez souligné ; pour s'en convaincre, il suffit de s'intéresser en général à l'évolution de la Convention de 2003, en particulier à ce que j'ai appelé la «prise française du PCI» (Tornatore 2011), et en détail au scandale que constitue selon moi la crypto-inscription de la gastronomie française sous l'appellation de « repas gastronomique des Français » et son actuelle concrétisation en Cités de la gastronomie dont les réalisations sont totalement tributaires de capitaux privés uniquement intéressés à vendre de l'image de marque. 


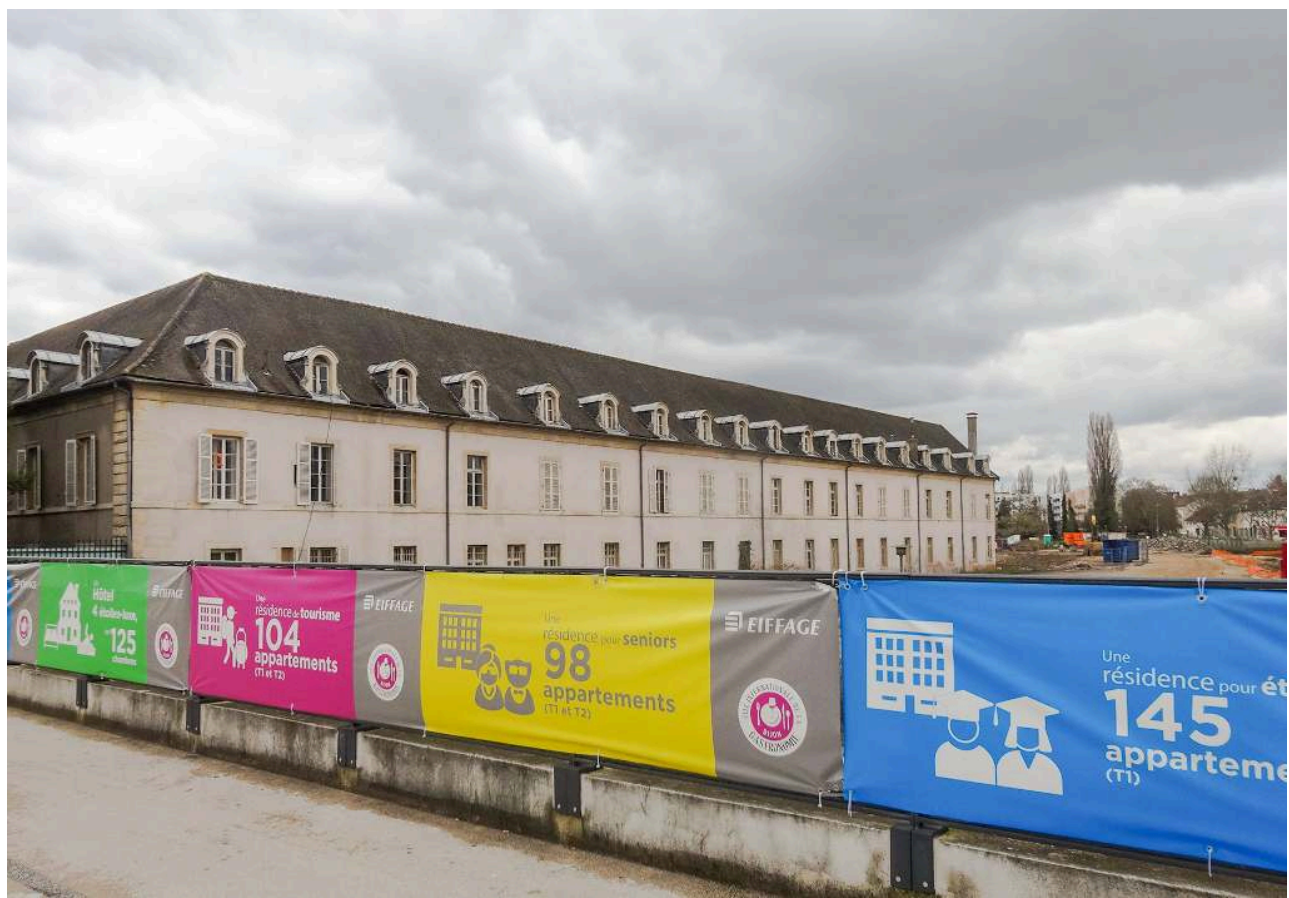

Figurant dans le cahier des charges de l'inscription du « Repas gastronomique des Français » sur la Liste représentative du patrimoine culturel immatériel (Unesco 2011), la Cité de la gastronomie se décline aujourd'hui en réseau de cités à Tours, Paris-Rungis, Lyon et Dijon. Confiée à un opérateur privé, le groupe Eiffage, celle de Dijon est actuellement en construction sur le site patrimonial de l'ancien hôpital général (MH 1908, 1930, 1937, 2007). Estimée à plus de 200 millions d'euros, l'opération a pour objectif la composition d'un site intégré et multifonctionnel alliant espaces culturels, centres de formations (à la cuisine française et aux vins), espace entrepreneurial, cinémas, hôtel de luxe dans la partie historique (concrétisant le lien entre luxe et patrimoine), restaurants et commerces, logements et résidences dans un nouvel « éco-quartier » En bref, la CIGV veut s'offrir comme une vitrine de l'excellence et du bien-vivre à Dijon, métropole patrimoniale et intelligente, où touristes et habitants seront invités à consommer, en toute quiétude et dans un esprit de divertissement la culture et le patrimoine local.

(c) Jean-Louis Tornatore.

19 3. Si la notion de "démocratie patrimoniale ", à laquelle j'ai pu naïvement adhérer (Tornatore 2017), a un sens, c'est-à-dire l'idée d'une action patrimoniale "d'en-bas », parallèle ou croisant les actions institutionnelles, c'est à condition de ne pas oublier de prendre en compte cette contrainte capitaliste et de considérer que des actions patrimoniales ont un potentiel non capitaliste (non lucratif, non possessif) ou anticapitaliste ${ }^{36}$.

4. Il ne peut y avoir de démocratie patrimoniale neutre, et encore moins, je l'ai déjà dit, de recherche neutre. On peut très bien se demander à l'infini et avec toujours plus de finesse - un pragmatisme très théorique, friand de décorticage philosophique semble s'y complaire - comment les gens agissent, ce qu'ils font et ce qui les fait faire, quelles sont les conséquences des concepts qu'ils utilisent dans l'action, quel est leur travail cognitif et évaluatif, il faut bien aussi se demander comment nous, chercheurs, scientifiques, anthropologues, agissons avec les gens qui agissent, ce que nous faisons avec les gens qui font. Le rejet du militantisme, c'est-à-dire de la confusion du chercheur et du militant, est trop ressassé pour ne pas être compris comme une forme de retour sans fin du dogme de la neutralité axiologique. Un chercheur dans son siècle n'a pas besoin d'être militant pour faire en sorte que les travaux et enquêtes qu'il 
conduit soient portés par et nourris de ses convictions éthiques et politiques; symétriquement, un militant n'a pas besoin d'être chercheur pour s'engager dans un travail de réflexion et d'analyse des situations - qu'il vit comme extérieures à lui -, la production écrite des militant.e.s et habitant.e.s des ZAD en est un éclatant exemple.

Patrimoine et anticapitalisme : habitation à la ZAD Notre-Dame-des-Landes.

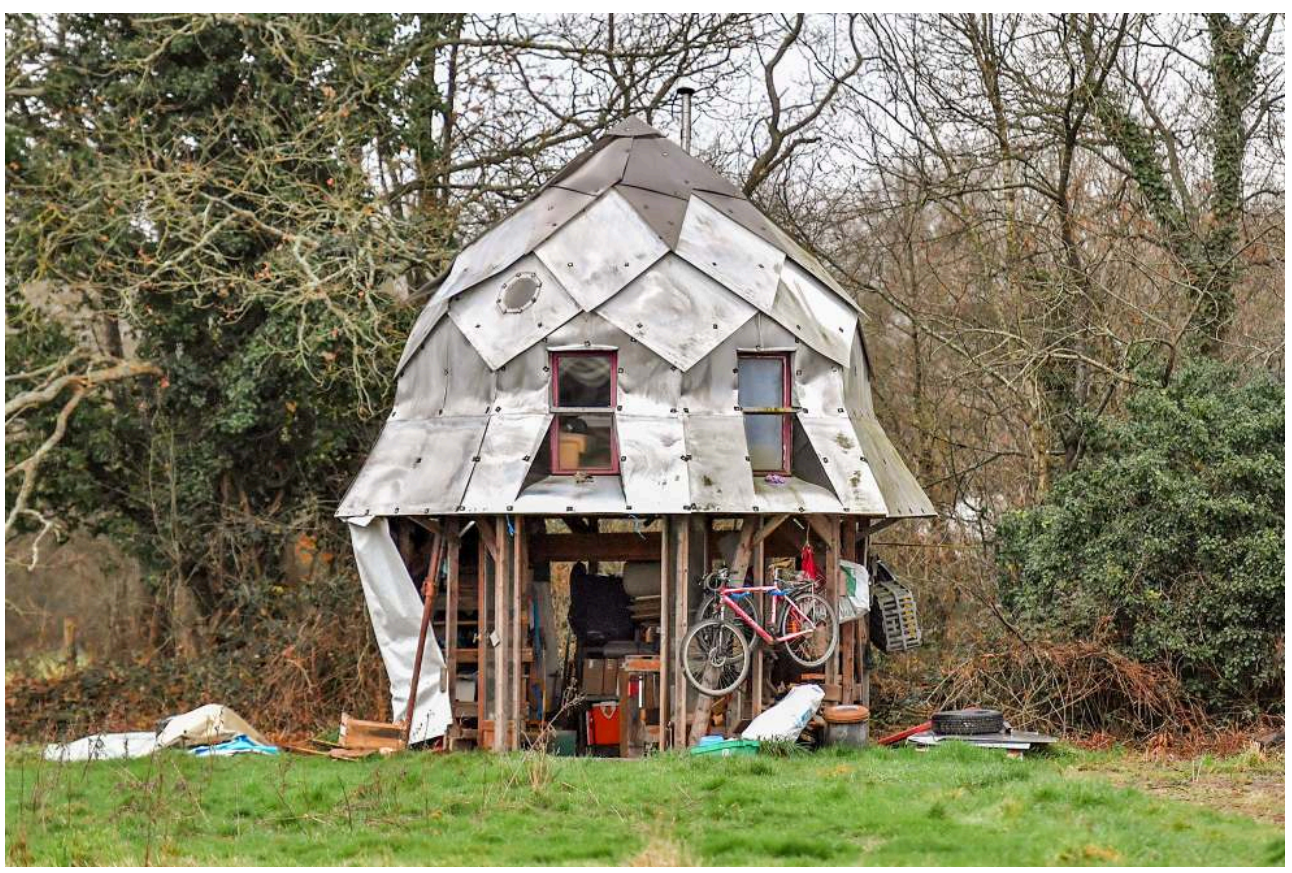

Le 18 mai 2018, alors que vient de débuter la deuxième vague de destruction et d'expulsions sur la ZAD de Notre-Dame-des-Landes, le collectif Défendre.habiter, réunissant des architectes, des paysagistes, des urbanistes, des habitant.es de la ZAD, saisit par courrier le Centre du patrimoine mondial de l'Unesco et l'alerte sur la nécessité urgente d'inscrire la ZAD et ses habitats sur la Liste du patrimoine mondial de l'humanité. Ce faisant, il attire l'attention sur la singularité des modes d'habiter en ce lieu, et sur le fait que peut-être s'y joue un sens nouveau, une conception nouvelle du patrimoine pour le XXI siècle : une manière de signifier ses attachements, de prendre soin, de faire attention auX choses et aux êtres humains et non-humains présents, pour le présent et pour le futur, de contribuer à une nouvelle manière d'être au monde. Cette habitation a été malheureusement détruite par les forces gouvernementales en mai 2018.

(C) Loïc Venance / cliché AFP.

21 C'est dans cette perspective que j'ai envisagé une interrogation sur la capacité du patrimoine à être mobilisé dans la construction de problèmes publics de notre temps. La liste est longue des situations vécues comme problématiques qui ont suscité et suscitent des "publics» et se traduisent en actions ou en mouvements sociaux: l'accaparement de biens publics par des entreprises privées, l'aménagementisme de l'État ou de collectivités territoriales, l'autoritarisme de plus en plus prégnant de l'État devant les situations dites " d'urgence ", son inertie, a contrario, face aux changements climatiques et à l'urgence écologique, l'incohérence des choix énergétiques, les destructions, les prédations et les injustices environnementales, les flux de populations et les tragédies des immigrations, l'uniformisation culturelle, la difficulté de vivre sa ou ses différences, le mépris toujours vivace pour les cultures populaires... Ces situations ou ces troubles peuvent être placés dans deux grands registres, étroitement liés : celui, d'une part, condensé par la dénomination proposée de notre époque, " anthropocène ${ }^{37}$ ", qui désigne la grande difficulté à penser un futur pour l'humanité et aiguise le sentiment de sa fragilité sur l'unique monde à sa disposition. On peut bien 
s'interroger sur le sort des monuments, des vestiges et des restes, sur la manière de les préserver, d'en prendre soin et d'y faire attention ${ }^{38}$, ces débats ne pèsent que peu de poids devant la perspective de plus en plus évidente que nous vivons dans un monde en ruine, sous menace d'effondrement (Servigne \& Stevens 2015). Celui, d'autre part, de l'expérimentation de formes nouvelles de la démocratie, de liens entre les êtres du monde, à partir de la conscience aigüe que la démocratie représentative est à bout de souffle et que les dispositifs politiques associés sont définitivement inopérants.

Que faire du patrimoine au regard de ces situations? Que fait le patrimoine? Quatre registres thématiques peuvent être explorés. Tout d'abord, le registre des actions patrimoniales citoyennes, c'est-à-dire de la refondation d'une pensée du patrimoine dans le cadre de mobilisations citoyennes relevant de la résolution de problèmes publics. La désignation de ce registre reste cependant éminemment fragile et suspecte, en raison de la charge de la notion de citoyenneté (par trop liée à l'État et au statut relatif aux populations administrées) et de son caractère attrape-tout ${ }^{39}$. En guise de substitution radicale, il serait peut-être plus juste, comme le suggère Noël Barbe, de parler d'actions "plébéiennes ", au sens exposé par Alain Brossat (2016), c'est-à-dire de ces actions qui relève du souci d'échapper au biopouvoir et du refus d'être gouverné et qui se déploient hors de la tutelle de l'État. Ensuite le registre d'une éthique du care et de l'attention élargie à tous les existants: non seulement, se préoccuper de lutter contre la dispersion de notre attention et la recentrer sur ce qui importe, le souci écologique comme le souci des autres (Citton 2014), mais s'intéresser aux formes d'action moins tournées vers la représentation du passé (au double sens de rendre présent et de présenter une nouvelle fois : définition canonique du patrimoine) que portées par le souci de faire attention à notre environnement présent ${ }^{40}$ et à notre monde pour les générations futures; cette logique du soin et de l'attention portés aux êtres et aux choses procédant de la volonté d'instruire une politique des attachements qui prenne le contrepied «du droit de ne pas faire attention » que s'est arrogé de capitalisme (Stengers 2009). Le registre, également, de l'inscription du patrimoine (comme de l'art ${ }^{41}$ ) dans la vie sociale ou ordinaire. Il ne s'agit pas seulement de reconnaître l'existence de patrimoines ordinaires ou populaires, distincts des patrimoines institutionnels, officiels, relevant de la culture légitime, mais également de considérer que le patrimoine irrigue la vie ordinaire, que les attachements relatifs au temps se jouent dans la vie ordinaire, et que la vie est à ce prix. Enfin, sous la reconnaissance des différences, le registre du "dissensus ", à partir duquel peuvent se construire des positions, toujours provisoires, de rencontre et de recherche de consensus qui ne résolvent pas pour autant les antagonismes. Il faut sortir de la vision lénifiante d'un patrimoine qui rapproche, établit des liants consensuels, lubrifie les rapports sociaux, sur fond de bons sentiments universalistes: les attachements certes relient ceux qui les partagent mais aussi séparent. 
Patrimoine et anthropocène 1 : Deep Time Closet, Mark Dion, 2001, exposition « Sublime. Les tremblements du monde », Centre Pompidou-Metz, 2016.

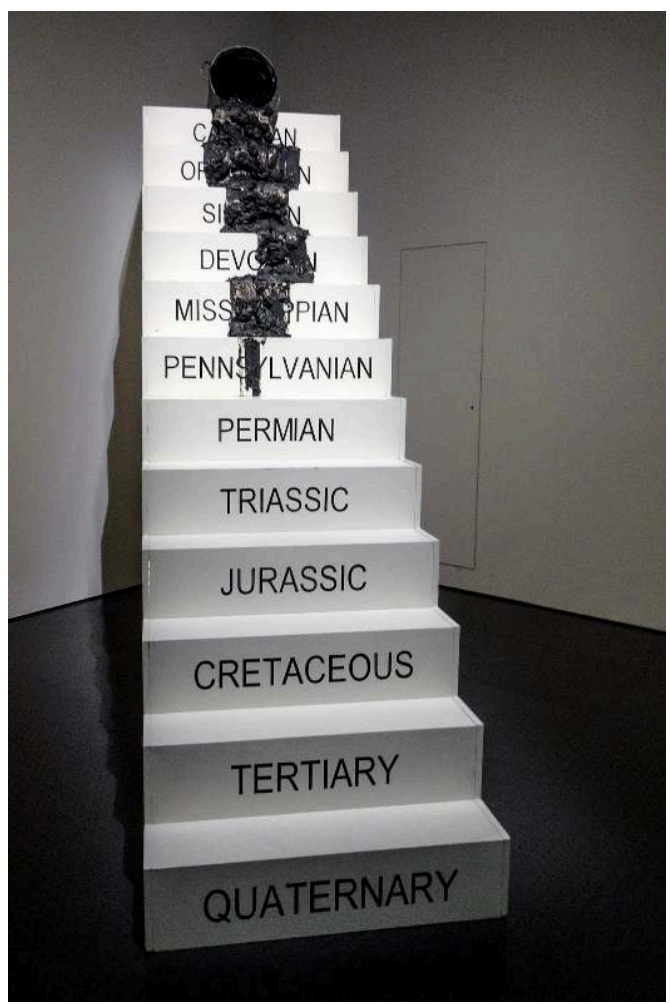

Contemporain de la popularisation par Paul Crutzen du terme " anthropocène », à partir de 2000, ce " placard du temps profond » est un hommage à Asphalt rundown (1969), œuvre de Paul Smithson, artiste qui dénonça très tôt les désastres causés par la civilisation industrielle. Le goudron qui s'écoule lentement sur les marches de l'échelle des temps géologiques constitue une nouvelle strate et le marqueur de l'action de l'humain, et plus précisément du capitalisme en tant qu'agent géologique. L'empreinte dévastatrice des énergies carbonées et l'extractivisme pèse sur le futur terrestre de l'espèce humaine et contribue à « retourner » la question patrimoniale : que devons-nous garder pour nous sauver, de quel patrimoine pouvons-nous nous réclamer pour vivre dans la perspective de l'effondrement?

(c) Jean-Louis Tornatore.

Ces registres constituent l'armature d'un scénario du patrimoine que j'appelle « contrehégémonique ", c'est-à-dire qui s'inscrit a contrario du modèle dominant du patrimoine, lequel est sous la double emprise de l'État et du marché. Ce scénario, il faut le préciser, ne répond pas à une logique de contre-pouvoir : par méfiance à l'égard de toute forme d'autorité inscrite dans un statut, et par intérêt pour les expérimentations de relations sociales fondées sur le refus d'exercer un quelconque pouvoir, il est fondamentalement anti-hégémonique. Ce modèle, on l'aura compris, exclut toute rupture entre recherche scientifique et action politique ${ }^{42}$. L'une est l'autre.

6. Question d'ontologies puis d'épistémologies. Un chantier important, dans le cadre de ce scénario contre-hégémonique, consiste à évaluer l'impact de ce que l'on a appelé le "tournant ontologique» de l'anthropologie ${ }^{43}$. Celui-ci prend de l'acuité devant la défaite de la nature (le monde naturel autant que son acception occidentale) et les limites de la notion anthropologique de culture. Pris dans les flux de cohortes touristiques de plus en plus impressionnantes, nous sommes devenus des visiteurs d'espaces protégés, réserves de culture comme réserves de nature. Nous sommes à l'orée du monde décrit dans Soleil vert ${ }^{44}$ et nous ne le voyons pas. Le tournant ontologique se déploie sur une double critique, critique de l'épistémologie, assimilée à 
la notion de représentation, et critique de la culture, assimilée à la notion de vision du monde : quant à la première, il avance l'idée d'une pluralité de mondes, quant à la seconde, l'idée d'une pluralité de réalités. Pour le dire rapidement, en laissant de côté les controverses qu'elle a suscitées ${ }^{45}$, une approche ontologique peut être congruente avec la perspective que nous développons en tant, en particulier, qu'elle renonce à l'opposition nature / culture, considère que la qualification des êtres n'est pas donnée à l'avance, est sensible aux enchevêtrements entre humains, choses et différentes formes de vie, fait entrer les êtres du vivant en politique et ouvre sur un débat cosmopolitique $^{46}$, interroge le rôle de l'anthropologue - décrire ou "contribuer activement à l'émergence de nouvelles formes d'attachements » (Keck, Regehr \& Walentowitz 2015 : 35). Plus généralement, la rencontre se fait dans le cadre d'une réévaluation de la relation au vivant : l'attachement ne se réalise pas dans une relation de domination, mais dans une relation de compagnonnage ou de partenariat, et configure une sorte de patrimoine compagnon comme il $\mathrm{y}$ a des espèces compagnes (Haraway 2010).

Patrimoine et Anthropocène 2 : Until MMXLI.IV, Darren Almond, 2002.

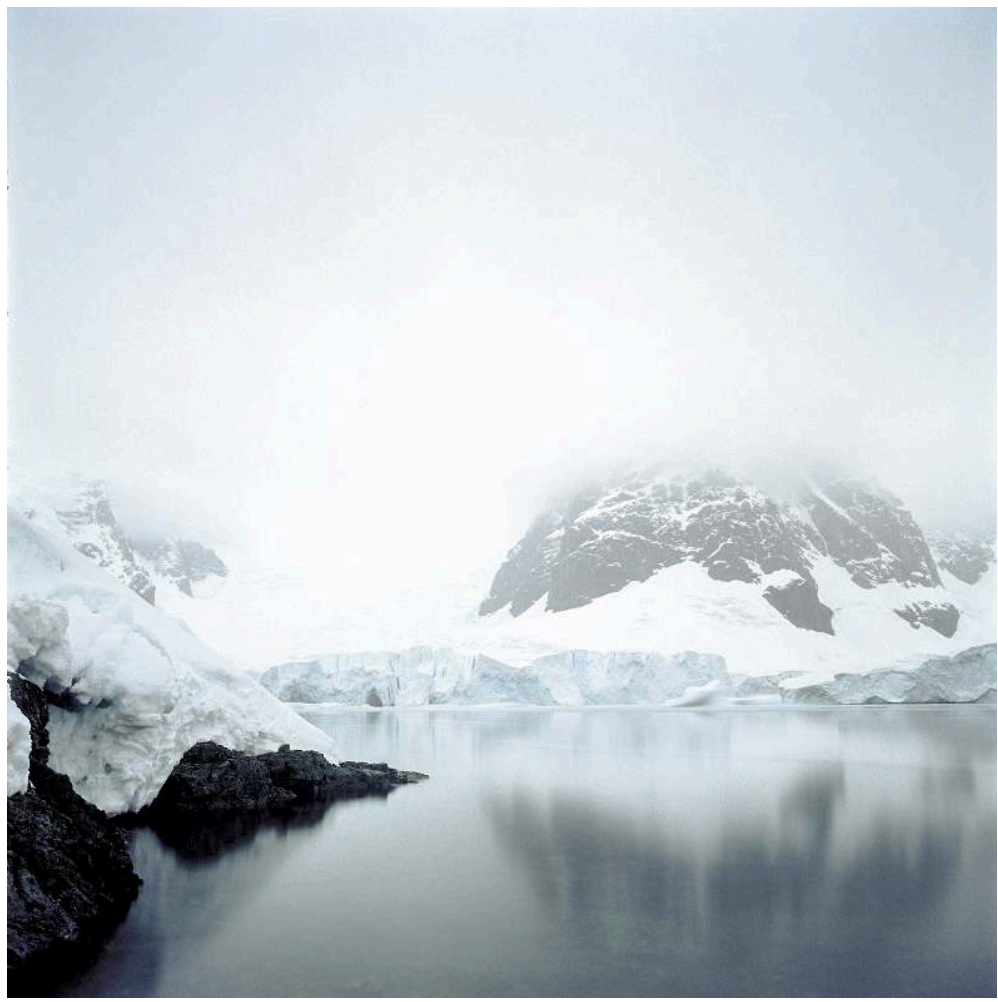

La série « Jusqu'en 2041 », de Darren Almond, rappelle l'échéance fixée par le « protocole de Madrid », amendement au Traité de l'Antarctique promulgué en 1991, validé pour cinquante ans, entré en vigueur en 1998, et désignant l'Antarctique comme « réserve naturelle consacrée à la paix et à la science ». Si elle souligne la nécessité d'une règlementation à même d'empêcher toute exploitation des ressources de ce territoire, elle rappelle que c'est là un des rares espaces de la Terre qui ne soit pas ou peu marqué par la présence humaine. Cadrant avec l'ontologie dualiste occidentale, l'Antarctique serait l'allégorie parfaite du « monde sans nous » - qui n'a jamais eu besoin de l'humain et qui doit le redouter.

(c) galerie Max Hetzler, Berlin.

La critique de l'épistémologie peut être déroutée en considérant que la négociation du rapport à la réalité dans une relation de connaissance n'est pas une exclusivité 
occidentale. Aussi l'approche ontologique faisant appel à des philosophies et à des ontologies non-occidentales doit utilement et nécessairement être complétée par l'exploration d'alternatives critiques et épistémologiques pointant les limites de la tradition critique occidentalocentriste et, dans un souci de justice, l'opportunité de l'ouvrir à d'autres modes de connaissance. Les travaux de Boaventura de Sousa Santos apportent sur ce point un éclairage majeur, arguant de la nécessité pour cette tradition critique d'" apprendre du Sud anti-impérialiste », le Sud étant considéré moins dans sa réalité géographique que comme "une métaphore de la souffrance humaine causée par le capitalisme et le colonialisme » (Santos $2011: 39$ ). Selon ce dernier, les possibilités de sortie du capitalisme et du colonialisme reposent sur trois conditions : considérer la grande diversité des rhétoriques et des scénarios visant des transformations émancipatrices; lier justice sociale et justice cognitive ; reconnaître les limites de la raison occidentale ${ }^{47}$ en ce qu'elle « ne peut accepter que la connaissance du monde soit plus grande que la compréhension occidentale du monde» (Santos 2016:27, 244). Prendre ses distances avec la tradition critique de manière à accueillir les " épistémologies du Sud » - à la fois de nouveaux savoirs valides, scientifiques ou non, et de nouvelles relations entre différents types de savoirs (Santos $2011: 38$ ) - nécessite la mise en œuvre d'une "sociologie des absences" et d'une "sociologie des émergences ", lesquelles mettent en question notre rapport univoque au temps et le gaspillage des expériences. Cette proposition renouvelle à mon sens puissamment la question du présentisme en ce qu'elle rend solidaires le présent et le futur dans une perspective émancipatrice. En effet, l'objectif de la sociologie des absences est d'étendre le présent à l'inexistant c'est-à-dire à ce qui est usuellement dénigré par la raison occidentale comme "ignorant, résiduel, inférieur, local et improductif » (Santos 2016 : 255). L'objectif de la sociologie des émergences est de contracter le futur: «le rendre rare et ainsi en faire un objet de soin » (ibid. : 266). Prendre soin du futur, c'est inscrire le futur (un futur incertain) dans le présent, c'est doter le présent de possibilités plurielles, à la fois utopiques et réalistes, en sorte que la contraction du futur contribue à l'expansion du présent (ibid.). Ainsi le présent devient riche à la fois d'expériences habituellement soustraites - considérées comme inexistantes - et de possibilités futures. Il est également enrichi de passés tout autant niés dans une tradition fondée sur la monoculture du temps linéaire et qui veut que «le passé [soit] automatiquement racheté par le futur» (ibid.: 132). A contrario, Santos avance la nécessité de « réinventer le passé comme négativité » (ibid.) de manière à lui « restituer sa capacité d'irruption et de rédemption» (ibid. : 131) et plaide pour "une autre conception du passé », un passé habité par «la souffrance humaine, l'exploitation et l'oppression", en sorte qu'il maintienne vif "notre non-conformisme et nos indignations " (ibid.). En somme, il y a des passés qui ne doivent jamais passer, dont on doit prendre soin, auxquels on doit donner un futur en ne les revoyant pas à l'inexistence ${ }^{48}$. La reconnaissance des épistémologies du Sud porte une mise en cause forte de nos politiques du passé et du patrimoine en tant qu'elles reposent sur la rupture du présent avec le passé et l'institution consécutive du "passé-comme-unautre $»^{49}$ et révèlent leur efficace dans trois fonctions : la neutralisation (réduire les aspérités sociales et les charges négatives) ${ }^{50}$, le volontarisme compensatoire (substituer la reconnaissance à la redistribution), la charité culturelle (reconnaître paternellement les invisibles et les inexistants) ${ }^{51}$. 


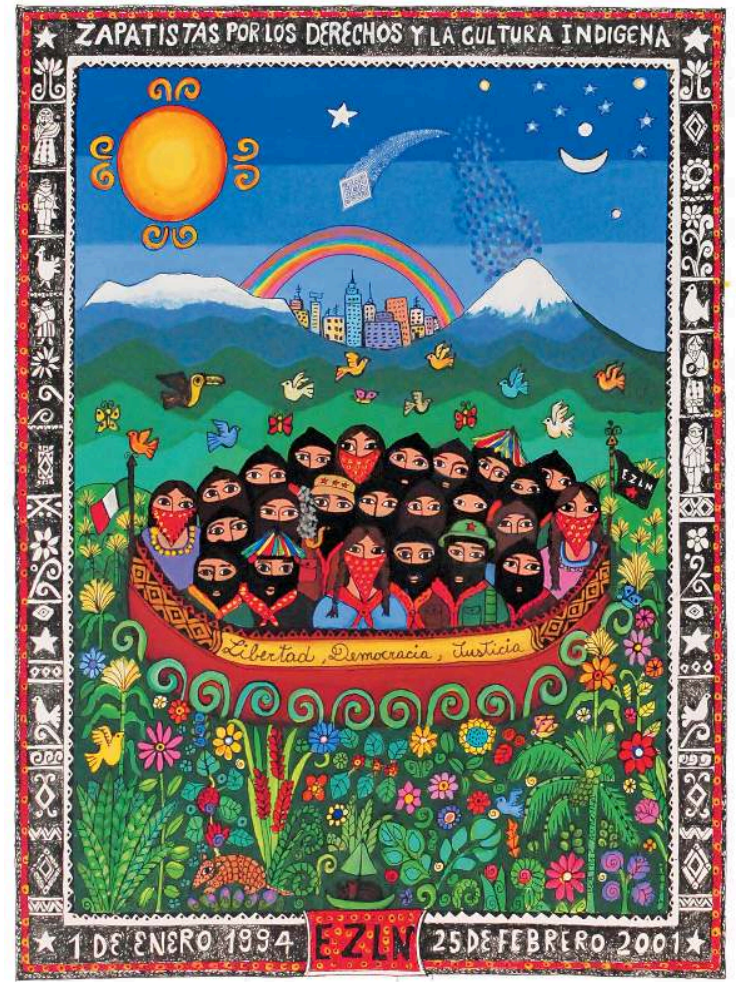

Ce tableau de l'artiste mexicaine Beatriz Aurora célèbre la marche pour la paix vers Mexico, initiée par le sous-commandant Marcos et l'Ejército zapatista de liberación nacional (EZLN) le 25 février 2001 -

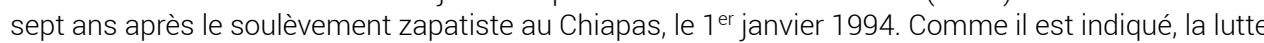
zapatiste pour « la liberté, la démocratie et la justice » passe par la reconnaissance des «droits et la culture indigène ». La perspective d'un futur dégagé de la domination capitaliste nécessite de lutter contre le présent perpétuel, de retrouver une conscience historique du passé et de reconnaître le temps cyclique et traditionnel des communautés, en un mot: de lutter pour la mémoire, contre l'oubli (voir Baschet 2018 : chapitre 1)

(c) Beatriz Aurora, www.beatrizaurora.blogspot.com.

En arguant de la nécessité de conditionner le futur à des passés présents, de Boaventura de Sousa Santos rejoint une idée importante formulée par Vinciane Despret (2013) ayant trait à la disqualification radicale de l'innocence. "Il faut ew $\mathrm{n}$ finir avec l'innocence » signifie qu'il faut refuser de dire " qu'on ne choisit pas ses ancêtres » et que, par conséquent, on n'est pas comptable du passé. Au contraire, choisir ses ancêtres, c'est hériter, c'est se déclarer responsable de ce dont nous héritons. Dans cette perspective, une pensée contre-hégémonique du patrimoine est une pensée de l'héritage, ce que la pensée hégémonique du patrimoine n'est paradoxalement pas dans la mesure où elle absout / innocente le passé. Si nous devons choisir nos ancêtres, c'est en pleine connaissance de causes au regard de tous les passés. Nous devons nous demander : qu'est-ce qu'hériter? De quoi héritons-nous ? De quoi pouvons-nous nous déclarer responsables? Nous avons à assumer tant d'héritages et de legs potentiels qui tous engagent des futurs incertains: des lieux de mémoire de mort, des espaces dégradés, des sites industriels abandonnés et pollués, des pertes irrémédiables (d'écosystèmes, d'espèces animales ou végétales), des déchets pullulants et des restes éternels, des gestes de destruction comme des gestes de sauvegarde, des résistances, des actions émancipatoires, des zones à défendre... En ce sens hériter, c'est instaurer des êtres qui comptent et savoir être comptable de ce que nous léguons. 
27 7. Question de transe. Dans un texte ambitieux, Daniel Cefaï (2010) a proposé une mise en perspective philosophique de l'expérience ethnographique, c'est-à-dire de l'engagement de l'ethnographe dans son métier, en s'appuyant sur la notion de mimèsis que Paul Ricœur a appliquée à l'analyse historiographique. Le schéma que ce dernier propose - la configuration narrative, autrement dit relative à la rédaction du texte historique, est encadrée en amont par un moment de préfiguration par les acteurs et en aval par un moment de refiguration par les lecteurs, ouvrant sur d'autres actions - est appliqué par Cefaï aux phases que l'ethnographe traverse dans son enquête : l'ancrage dans le monde des enquêtés, la transmutation de cette expérience en texte ethnographique, la réception et la reprise de l'enquête sur le terrain. Il se propose ainsi de «réinterroger le problème de la normativité à l'œuvre dans les multiples engagements de l'ethnographe, mais tout en tenant à distance les raccourcis activistes, pour qui les sciences sociales ne sont qu'une autre manière de faire de la politique » (ibid. : 548-549, c'est moi qui souligne). Il confirme plus loin qu'après la coopération et l'implication vient un nécessaire détachement vis-à-vis du monde des acteurs. S'il convient de la pluralité des modes d'engagement, de l'«incroyable diversité des contextes d'investigation » (la vie familiale, la vie scientifique, le travail social, l'aide humanitaire, la mondialisation, les luttes des peuples autochtones, les catastrophes sanitaires et écologiques, les pratiques médicales clandestines, le monde des dealers et de la toxicomanie, etc.) impliquant chaque fois des modalités singulières d'engagement, de l'empathie à la médiation et au conseil, et que finalement, "il n'y a pas de règle générale que l'ethnographe puisse suivre » (ibid. : 594), il estime qu'il existe un seuil que le «pragmatisme ethnographique » ne doit pas franchir, celui du militantisme : s'il ne renonce pas à la critique, l'ethnographe «ne poursuit pas avec d'autres armes un combat de classe, de genre ou de race» (ibid. 593), "il n'a pas être du côté des “dominés", ni de celui des "dominants", ni des "vainqueurs" ni des "vaincus", dans la mesure où il s'efforce avant tout de rendre compte de la complexité des relations sociales » (ibid.) ; il « doit retenir ses jugements de valeur » (ibid.) parce que « la liberté par rapport aux valeurs est un engagement de tous les instants au service d'une vérité [...] afin de rendre disponible à des auditoires, plus ou moins intéressés, autorisés et spécialisés des éléments d'information qui, si complexes soient-ils l'aident à se former une opinion et un jugement » (ibid. : 594).

Cefaï reconnaît - «ces préceptes-là ne nous semblent pas caducs » (ibid.: 593) - se situer dans une tradition intellectuelle, sociologique et anthropologique, que l'on peut dire nourrie au biberon de la neutralité et de la dialectique de la proximité et de la distance. C'est ici, me semble-t-il, que peut se jouer a contrario une rencontre forte de l'anthropologie et du pragmatisme. Le seul moyen de transgresser la dialectique de la proximité et de la distance que les anthropologues ont trouvée a été de doubler leurs textes scientifiques par des textes d'ambition littéraire. Vincent Debaene (2010) a magistralement analysé le cas notable d'ethnologues français de renom (ceci expliquant aussi cela) qui, de retour de leur terrain, ont écrit non pas un livre, mais deux, l'un scientifique l'autre littéraire, le second venant combler le manque qu'a généré le premier en gommant l'expérience subjective de dépaysement, de dépouillement de soi, d'arrachement, que tout ethnographe est censé vivre dans et avec son corps (Debaene 2006). En même temps la tradition anthropologique a glosé sur un nécessaire geste de retrait ou de retenue, sous peine pour l'ethnologue de perdre sa raison et son identité, et l'ethnologie son projet scientifique ${ }^{52}$. Pour autant, si le temps de l'écriture est celui du « contact » perdu au moyen des concepts puis retrouvé par le 
style réintroduisant les percepts et les affects, celui du terrain, poussé à une sorte de paroxysme, reste l'épreuve reine qu'il faut à la fois vivre pleinement et sans retenue, et dont il faut à un moment donné, littéralement, s'arracher. Si je me réfère à ma propre expérience, il existe dans le temps du terrain un moment de basculement par lequel je me semble accéder à l'autre en m'identifiant à lui : un état de transe ${ }^{53}$, si bref soit-il, au cours duquel je fais l'expérience de passer de l'autre côté et d'être l'autre. Si, en ce sens, l'ethnographie est un "art de transe » ou "de lâcher-prise " ${ }^{54}$, celui-ci reste vécu a posteriori sur le mode de la culpabilité - enfreindre les règles de la raison scientifique -, de l'impuissance à terme due à l'aveuglement procuré par la vision de (trop) près, et de l'angoisse du non-retour ou d'une sorte de mauvais trip dont on redescendrait quelques neurones en moins. Or, un des premiers apports du pragmatisme a été de défaire l'inquiétude d'être trop près pour bien voir et bien dire ce que l'on voit, de ne pas être à la hauteur, à la bonne distance de ce que les gens font, en recommandant de descendre de son nuage épistémologique et de les suivre, moins déterminés qu'agissant en toute incertitude et mobilisant des ressources dans l'action, d'observer au ras des situations le social en train de se faire. Tout se passe comme si Cefaï retenait le pas en avant, décisif, accompli par le pragmatisme sociologique dont il se veut être un représentant. En restreignant l'attitude pragmatiste au temps du terrain, il vide cette posture de sa capacité de subversion radicale de la démarche et de la fonction de recherche scientifique. La singularité et la force du pragmatisme tiennent dans la proposition de se déprendre ${ }^{55}$ des formatages, les dualismes en particulier, qui ont crû sur le terreau accueillant du rationalisme académique ; il est un vecteur d'émancipation et doit se vivre comme un devenir. Pour continuer la métaphore du passage, le devenir-pragmatiste serait un art, ici achevé, de transe: invitant à rester de l'autre côté, à y être soi avec l'autre.

S'il s'agit de réinventer avec le pragmatisme une autre manière de faire science, on peut remarquer, au regard des terrains de militantisme cités par Daniel Cefaï, tels la classe, le genre ou la race, qu'il est vain de trouver des cas de figure desquels le chercheur, qu'il soit homme, femme ou transgenre, blanc, noir, jaune, rouge ou vert, issu d'un milieu populaire, de bonne famille ou entre les deux, soit totalement extérieur. En ce sens, à moins du cas devenu improbable de rencontrer une population jamais " contactée ", comme disent les américanistes et les océanistes, passer de l'autre côté c'est toujours rester chez soi, en sorte que les différences se révèlent dans la proximité. Cela est d'autant plus vrai si l'on se sent concerné par l'empoisonnement du monde par le capitalisme: en ce cas, il n'y a pas à être "du côté de " puisqu'on considère qu'on est déjà, tous ensemble, dans la panade jusqu'au cou et que toutes les ressources sont bonnes, celle de la recherche scientifique comme d'autres, pour essayer de s'en sortir. 


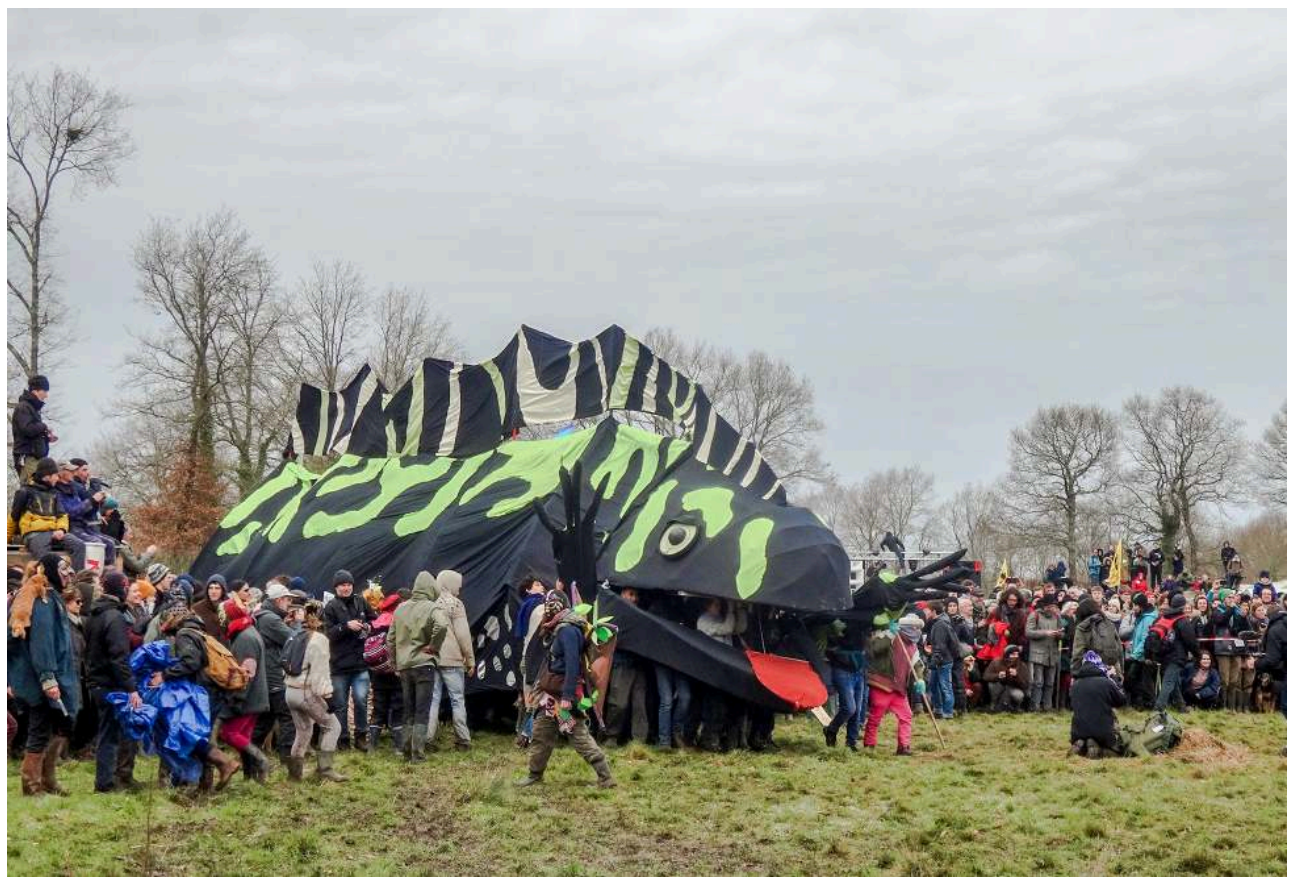

Le triton crêté, espèce protégée et familière du bocage, est peut-être devenu le « totem » de la ZAD Notre-Dame-des-Landes, au moins le temps de la Fête de la victoire, célébrant l'abandon du projet d'aéroport, le 10 février 2018 et qui a réuni plus de 20000 personnes. Joyeusement porté par plusieurs dizaines de personnes, il s'engage, avec d'autres totems représentant d'autres ZAD, dans une farandole endiablée autour d'un grand avion de bois et de paille qui finira par s'embraser. Tout compte fait, la convivialité, le partage, et bien d'autres valeurs encore - attention, soin, attachement, mise en commun(s)... - sont portés par les ZAD et autre expériences qui laissent entrevoir qu'il est possible non de changer le monde mais de changer de monde - à tout le moins, que d'autres mondes sont possibles.

(c) Jean-Louis Tornatore.

8. Question, encore, de désignation. Le patrimoine peut être vu comme une modalité spécifique d'attachement. Et s'il est vrai que les êtres qu'il définit sont des partenaires dans les interactions sociales, ils le sont par deux fois : au titre de choses avec lesquelles nous interagissons, au titre de la qualité patrimoniale dont on les dote. Ainsi, ce sont des êtres qui comptent doublement. En ce sens le patrimoine est une institution de l'importance. La question qui court en sourdine tout au long de ce texte est qu'elle a peut-être fait son temps sous cette désignation. Ellen Hertz et Susanne ChappazWirthner (2012) l'ont déjà posée mais, arguant que « le concept de patrimoine doit être laissé aux usagers ordinaires et exclu du langage scientifique », pour des raisons qui vont à l'opposé du pragmatisme. Dans les perspectives que j'ai suggérées, une anthropologie pragmatiste et plébéienne du patrimoine - tout comme d'ailleurs une anthropologie pragmatiste de la citoyenneté - doit se dégager de l'emprise du formatage étatique, toujours et difficile à dénouer. Je balance donc entre deux solutions : soit laisser le mot à l'État et au Marché et en promouvoir un autre «non-préoccupé », qui, tel "attachement ", pourrait être pleinement approprié à la posture inclusive et politique que nous souhaitons promouvoir; soit refuser de l'abandonner ${ }^{56}$ et travailler à le doter de nouvelles relations de signification. Sans doute, en proposant une diversité d'entrées, ce texte est-il la parfaite expression de la seconde. Aussi je terminerai par la proposition d'une nouvelle entrée avec un mot qui serait aussi un possible candidat à la substitution et qui, en même temps, est à même d'enrichir (au 
sens ici d'augmenter) les dimensions à la fois pratiques et conceptuelles de "patrimoine » : une politique du ou des patrimoines se recomposant dans une politique du ou des «communs». Pierre Dardot et Christian Laval (2014) en font un principe central de notre siècle au croisement de la lutte anticapitaliste et de l'écologie politique ; afin de le prémunir contre toute récupération par l'appareil technocratique d'État, Pascal Nicolas-Le Strat (2016:63 sq.) insiste sur le caractère « oppositionnel » du travail du commun. On voit combien le mot est susceptible d'éclairer la perspective alternative, radicale, contre-hégémonique (anticapitaliste) d'une pensée des attachements à l'Anthropocène. Question de commun(s) : un chantier à venir.

\section{BIBLIOGRAPHIE}

ANDERSON Bénédict, 1996, L'Imaginaire national : réflexions sur l'origine et l'essor du nationalisme, Paris : La Découverte.

BARBE Noël (dir.), 2003, « Actions patrimoniales », Les Nouvelles de l'archéologie, n 93, p. 5-7.

BARBE Noël, 2013, « Isac Chiva, ethnologie et politique patrimoniale », Terrain, $n^{\circ} 60$,

«L'imaginaire écologique », p. 148-163. Disponible en ligne, http://journals.openedition.org/ terrain/15127 [lien valide en juin 2018].

BARBE Noël, CHAULIAC Marina \& ToRNATORE Jean-Louis, 2015, « Intangible cultural heritage exposed to public deliberation :

a participatory experience in a Regional Nature Park ", in ADELL Nicolas, BENDIX Regina F., BORTOLOTTO Chiara, TAUSCHEK Markus (dir.), Between imagined communities and communities of practice, Göttingen : Universitätsverlag Göttingen, « Göttingen Studies in Cultural Properties », p. 201-2108.

BARTHE Yannick et al., 2013, «Sociologie pragmatique : mode d'emploi », Politix, $\mathrm{n}^{\circ} 103$, « Représenter les agriculteurs », p. 175-204. Disponible en ligne, https://www.cairn.info/revuepolitix-2013-3-page-175.htm [lien valide en juin 2018].

BASCHET Jérôme, 2018, Défaire la tyrannie du présent. Temporalités émergentes et futurs inédits, Paris, La Découverte, coll. « L'horizon des possibles».

BENATOUïL Thomas, 1999, «Critique et pragmatique en sociologie. Quelques principes de lecture ", Annales. Histoire, sciences sociales, vol. 54, n² 2, p. 281-317. Disponible en ligne, https:// www.persee.fr/doc/ahess_0395-2649_1999_num_54_2_279749 [lien valide en juin 2018].

BIDET Alexandra, QUÉRÉ Louis \& TRUC Gérôme, 2011, « Ce à quoi nous tenons. Dewey et la formation des valeurs », in DEWEY John, La Formation des valeurs, Paris : La Découverte, coll. « Les empêcheurs de penser en rond ", p. 5-64.

BOLTANSKI LUc, ESQUERRE Arnaud, 2017, Enrichissement. Une critique de la marchandise, Paris : Gallimard, coll. « NRF Essais ».

BONNEUIL Christophe, FRESSOZ Jean-Baptiste, 2013, L'Événement anthropocène. La Terre, l'histoire et nous, Paris : Éditions du Seuil, coll. « Anthropocène ». 
BORTOLOTTо Chiara, 2015, « Unesco and heritage self-determination : Negotiating meaning in the Intergovernmental Committee for the Safeguarding of the ICH », in ADELL Nicolas, BENDIX Regina, BORTOLOTTO Chiara, TAUSCHEK Markus (dir.), Between imagined communities and communities of practices. Participation, territory and the making of heritage, Göttingen, Universitätsverlag Göttiengen, coll. « Göttingen Studies in Cultural Property », p. 249-272.

BRosSAT Alain, 2016, "Pour une politique plébéienne », Ici et Ailleurs. Association pour une philosophie nomade [blog], https://ici-et-ailleurs.org/voyons-ou-la-philo-mene/article/pour-unepolitique-plebeienne [lien valide en juin 2018].

BRUMANN Christoph, 2014, « Heritage agnosticism. A third path for the study of cultural heritage ", Social Anthropology / Anthropologie sociale, vol. 22, n², p. 173-188.

CALlon Michel, LASCOUMES Pierre \& BARTHE Yannick, 2001, Agir dans un monde incertain. Essai sur la démocratie technique, Paris, Éditions du Seuil, coll. « La couleur des idées ».

CAMPBELl Gary \& SMITH Laurajane, 2016, « Keeping critical heritage studies critical. Why "posthumanism" and the "new materialism" are not so critical ", conférence inédite prononcée au cours de la $3^{\mathrm{e}}$ conférence biennale de l'Association of Critical Heritage (Montréal, 3-8 juin 2016), «What does heritage change?».

CARBonell Charles-Olivier, 1978, « L'histoire dite "positiviste” en France », Romantisme, n 21-22, « Les positivismes », p. 173-185. Disponible en ligne : https://www.persee.fr/doc/ roman_0048-8593_1978_num_8_21_5216 [lien valide en juin 2018].

CARRITHERS Michael, CANDEA Matei, sYKes Karen, HOLBRAAD Martin \& VENKATESAN SOUMHYA, 2010, « Ontology is just another word for culture. Motion tabled at the 2008 Meeting of the Group for Debates in Anthropological Theory, University of Manchester ", Critique of Anthropology, vol. $30, n^{\circ} 2$, p. $152-200$.

CEFAÏ Daniel, 2010, « L'expérience ethnographique, l'enquête et ses publics », in CEFAÏ Daniel (dir.), L'Engagement ethnographique, Paris, Éditions de l'EHESS, coll. « En temps \& lieux », p. 547-597.

CEFAÏ Daniel, 2016, « Publics, problèmes public, arènes publiques... Que nous apprend le pragmatisme?», Questions de communication, $\mathrm{n}^{\circ} 30, \mathrm{n}^{\circ}$ 2, p. 25-64. Disponible en ligne : https:// journals.openedition.org/questionsdecommunication/10704 [lien valide en juin 2018].

CHARLE Christophe, 2011, Discordance des temps. Une brève histoire de la modernité, Paris, Armand Colin, coll. « Le temps des idées ».

CHARTIER Denis \& RODARY Estienne (dir.), 2015a, Manifeste pour une géographie environnementale. Géographie, écologie, politique, Paris, Presses de Sciences Po.

CHARTIER Denis \& RoDARY Estienne, 2015b, « Géographie, écologie, politique. Un climat de changement », in CHARTIER Denis, RODARY Estienne (dir.), Manifeste pour une géographie environnementale. Géographie, écologie, politique, Paris, Presses de Sciences Po, p. 13-56.

Chateauraynaud Francis \& cohen Yves (dir.), 2016, Histoires pragmatiques, Paris, Éditions de l'EHESS, coll. « Raisons pratiques ».

CITTon Yves, 2014, Pour une écologie de l'attention, Paris, Éditions du Seuil, coll. « La couleur des idées" ".

COMITÉ INVISIBLE, 2017, Maintenant, Paris, La Fabrique éditions. 
COUTELLEC Léo, 2015, La Science au pluriel. Essai d'épistémologie pour les sciences impliquées, Versailles, Éditions Quae, coll. «Sciences en questions ».

DARDoT Pierre \& LAVAL Christian, 2014, Commun. Essai sur la révolution au XIXe siècle, Paris La Découverte.

DEBAENE Vincent, 2006, « “Étudier des états de conscience.” La réinvention du terrain par l'ethnologie, 1925-1939 », L'Homme, n 179, « Des raisons du terrain », p. 7-62. Disponible en ligne : https://journals.openedition.org/lhomme/24033 [lien valide en juin 2018].

DEBAENE Vincent, 2010, L'Adieu au voyage. L'ethnologie française entre science et littérature, Paris, Gallimard, coll. « Bibliothèque des sciences humaines ».

DEBAISE Didier \& STENGERS Isabelle (dir.), 2015, Gestes spéculatifs, actes du colloque de Cerisy (28 juin-5 juillet 2013), Dijon, Les Presses du réel, coll. « Drama ».

DEBAISE Didier, 2015, «L'intensification de l'expérience », in DEBAISE Didier, STENGERS Isabelle (dir.), Gestes spéculatifs, actes du colloque de Cerisy (28 juin-5 juillet 2013), Dijon, Les Presses du réel, coll. « Drama », p. 106-120.

DELACRoIX Christian, dosSE François \& GARCiA Patrick, 1999, Les Courants historiques en France, $19^{e}-20^{e}$ siècles, Paris, Armand Colin, coll. «U », série « Histoire ».

DELEUZE Gilles \& PARNET Claire, 1996, Dialogues, Paris, Flammarion, coll. « Champs ».

DESPRET Vinciane, 2013, « En finir avec l'innocence. Dialogue avec Isabelle Stengers et Vinciane Despret », in DoRLIn Elsa, Rodriguez Eva (dir.), Penser avec Donna Haraway, Paris, PUF, coll. « Actuel Marx confrontation », p. 22-45.

DEWEY John, 1967 [1938], Logique. La théorie de l'enquête, Paris, PUF.

DEWEY John, 2003 [1927], Cuvres philosophiques, tome 2, Le Public et ses problèmes, Pau / Tours / Paris, Presses universitaires de Pau / Farrago / Éditions Léo Scheer.

DEWEY John, 2011 [1939], La Formation des valeurs, Paris, Les empêcheurs de tourner en rond / La Découverte.

DEWEY John, 2012a [1905], « Le postulat de l'empirisme immédiat », Critique, n 787, vol. 12, « Retour à Dewey », p. 1014-1025. Disponible en ligne : https://www.cairn.info/revuecritique-2012-12-page-1014.htm [lien valide en juin 2018].

DEWEY John, 2012b [1925], Expérience et Nature, Paris, Gallimard, coll. « Bibliothèque de philosophie ".

DIANTEILL Erwan \& HELL Bertrand (dir.), 2008, Gradhiva, n 7, « Le possédé spectaculaire. Possession, théâtre et globalisation ».

ESCOBAR Arturo, 2018, Sentir-penser avec la terre. L'écologie au-delà de l'Occident, Paris, Éditions du Seuil, coll. « Seuil Anthropocène ».

FABRE Daniel (dir.), 2013, Émotions patrimoniale, Paris, Éditions de la Maison des sciences de l'homme / ministère de la Culture et de la Communication, coll. « Cahiers d'ethnologie de la France ».

FABRE Daniel, 1994, «"Ethnologie et patrimoine en Europe." Conclusions et perspectives du colloque de Tours », Terrain, n²2, «Les Émotions », p. 145-150. Disponible en ligne : https:// journals.openedition.org/terrain/3095 [lien valide en juin 2018]. 
FABRE Daniel, 2014, « La pérennité », in HEINICH Nathalie, SCHAEFFER Jean-Marie, TALON-HUGON Carole (dir.), Par-delà le beau et le laid. Enquêtes sur les valeurs de l'art. Rennes, Presses universitaires de Rennes, coll. « Aesthetica », p. 83-104.

FABRE Daniel, 2016, « L'ordinaire, le familier, l'intime, loin du monument », in HоTтIN Christian, voISENAT Claudie (dir.), Le Tournant patrimonial. Mutations contemporaines des métiers du patrimoine, Paris, Éditions de la Maison des sciences de l'homme / ministère de la Culture et de la Communication, coll. « Cahiers d'ethnologie de la France », p. 43-58.

FRESSOZ Jean-Baptiste, GRABER Frédéric, LOCHER FABIEN \& QUENET Grégory, 2014, Introduction à l'histoire environnementale, Paris, La Découverte, coll. « Repères », série « Histoire ».

FUREIX Emmanuel \& JARRIGE François, 2015, La Modernité désenchantée. Relire l'histoire du XIXe siècle français, Paris, La Découverte, coll. « Écritures de l'histoire ».

GROTOWSKI Jerzy, 1971 [1968], Vers un théâtre pauvre, Lausanne, La Cité / L’Âge d'Homme, coll. « Théâtre vivant ».

Guillaume Marc, 1980, La Politique du patrimoine, Paris, Galilée, coll. « L'espace critique ».

HACHE Émilie, 2011, Ce à quoi nous tenons. Propositions pour une écologie pragmatique, Paris, La Découverte / Les empêcheurs de tourner en rond.

HARAWay Donna J., 2009, « Savoirs situés : la question de la science dans le féminisme et le privilège de la perspective partielle", in Des singes, des cyborgs et des femmes. La réinvention de la nature, Paris / Arles, Jacqueline Chambon / Actes Sud, coll. « Rayon philo », p. 323-352.

HARAWay Donna J., 2010, Manifeste des espèces de compagnie. Chien, humains et autres partenaires, Éditions de l'Éclat, coll. « Terra cognita ».

HARRISON Rodney, 2013, Heritage. Critical approaches, Londres, Routledge.

HARTOG François, 2003, Régimes d'historicité. Présentisme et expériences du temps, Paris, Éditions du Seuil, coll. « La librairie du XXI ${ }^{\mathrm{e}}$ siècle ».

HARVEY Davis, 2008, Géographies de la domination, Paris, Les Prairies ordinaires, coll. « Penser/ croiser ».

HENNION Antoine, 2010, « Vous avez dit attachements ?... », in AKRICH Madeleine, BARTHE Yannick, MUNIESA Fabian, MUSTAR Philippe (dir.), Débordements. Mélanges offerts à Michel Callon, Paris, Presses de l'École des Mines, coll. « Sciences sociales », p. 179-190.

HENNION Antoine, 2015, " Enquêter sur nos attachements. Comment hériter de William James?", Sociologies [en ligne], « Pragmatisme et sciences sociales : explorations, enquêtes, expérimentations », http://sociologies.revues.org/4953 [lien valide en juin 2018].

HERTZ Ellen \& CHAPPAZ-WIRTHNER Suzanne, 2012, « Introduction : le "patrimoine" a-t-il fait son temps? », Ethnographiques.org [en ligne], $n^{\circ} 24$, « Ethnographies des pratiques patrimoniales : temporalités, territoires, communautés ", http://www.ethnographiques.org/2012/HertzChappaz-Wirthner [lien valide en juin 2018].

HERTZ Ellen, 2015, « Bottoms, genuine and spurious », in ADELL Nicolas, BENDIX Regina, BORTOLOTTO Chiara \& TAUSCHEK Markus (eds), Between Imagined Communities and Communities of Practices. Participation, Territory and the Making of Heritage, Göttingen, Universitätsverlag Göttiengen, p. 25-57.

JAMES William, 2005 [1912], Essais d'empirisme radical, Marseille, Agone, coll. « Banc d'essais ». 
JAMES William, 2007 [1909], Philosophie de l'expérience. Un univers pluraliste, Paris, Éditions du Seuil / Les Empêcheurs de penser en rond.

JARRIGE François, 2014, Technocritiques. Du refus des machines à la contestation des technosciences, Paris, La Découverte.

KALINOWSKI Isabelle, 2005, « Leçons wébériennes sur la science et la propagande », in WEBER Max, La Science, profession et vocation, Marseille, Agone, coll. « Banc d'essais », p. 61-273.

KECK Frédéric, REGEHR Ursula \& WALENTOWITZ Saskia, 2015, « Anthropologie : le tournant ontologique en action. Introduction ", Tsantsa, $\mathrm{n}^{\circ} 20$, « L'anthropologie et le tournant ontologique », p. 34-41. Disponible en ligne, https://tsantsa.ch/fr/archives/numero-20/ contenu-20-2015/dossier/anthropologie-le-tournant-ontologique-en-action [lien valide en juin 2018].

KOSELLECK Reinhardt, 1997, L'Expérience de l'histoire, Paris, Gallimard / Éditions du Seuil, coll. « Hautes études».

LAFERTÉ Gilles \& RENAHY Nicolas, 2003, «L'ethnologue face aux usages sociaux de l'ethnologie », L'Homme, $n^{\circ} 166$, « Malinowski, Faulkner. Culture et cognition. Souvenir et héritage », p. 239-240. Disponible en ligne, https://journals.openedition.org/lhomme/229 [lien valide en juin 2018].

LANDIVAR Diego \& RAMILLIEN Émilie, 2015, « Reconfigurations ontologiques dans les nouvelles constitutions andines. Une analyse anthropologique, Tsantsa, $n^{\circ} 20$, « L'anthropologie et le tournant ontologique », p. 34-44. Disponible en ligne, https://tsantsa.ch/fr/archives/numero-20/ contenu-20-2015/dossier/reconfigurations-ontologiques-dans-les-nouvelles-constitutionspolitiques-andines [lien valide en juin 2018].

LATOUR Bruno, 2000, « Factures / fractures : de la notion de réseau à celle d'attachement », in MICOUD André, PERONI Michel (dir.), Ce qui nous relie, La Tour d'Aigues, Éditions de l'Aube, coll. « Société et territoire », p. 189-207.

LATour Bruno, 2012, Enquête sur les modes d'existence. Une anthropologie des modernes, Paris, La Découverte.

LEIGH STAR Susan \& GRIESEMER James R., 1989, « Institutional ecology, "translations" and boundary objects. Amateurs and professionals in Berkeley's Museum of Vertebrate Zoology, 1907-1939 ", Social Studies of Science, vol. 19, p. 387-420.

LEIGH STAR Susan, 2010, « “Ceci n'est pas un objet-frontière !” Réflexions sur l'origine d'un concept », Revue d'anthropologie des connaissances, vol. 4, $\mathrm{n}^{\circ}$ 1, p. 18-35.

LEPETIT Bernard (dir.), 1995, Les Formes de l'expérience. Une autre histoire sociale, Paris, Albin Michel, coll. «L'évolution de l'humanité ».

MÉRIMÉE Prosper, 1998 [1934], La Naissance des monuments historiques. La correspondance de Prosper Mérimée avec Ludovic Vitet (1840-48), Paris, Éditions du CTHS.

MONDZAIN Marie José, 2017, Confiscation : des mots, des images et du temps, Paris, Éditions Les liens qui libèrent.

Mosès Stéphane, 2006, L'Ange de l'Histoire. Rosenzweig, Benjamin, Scholem, Paris, Gallimard, coll. « Folio », série « Essais».

MULTITUDES, 2017, $\mathrm{n}^{\circ}$ 66, vol. 1, « Ceci n'est pas un programme ». 
NEVEU Catherine, 2005, Anthropologie de la citoyenneté, mémoire d'Habilitation à diriger des recherches, université de Provence - Aix-Marseille I. Disponible en ligne, https://tel.archivesouvertes.fr/tel-00627444/document [lien valide en juin 2018].

NICOLAS-LE STRAT Pascal, 2016, Le Travail du commun, Saint-Germain-sur-Ille, Éditions du commun. OFFENSTADT Nicolas \& VAN DAMME Stéphane, 2009, « Les pratiques historiennes au risque de la sociologie pragmatique. Trajectoires et perspectives ", in BREVIGLIERI Marc, LAFAYE Claudette \& TROM Danny, Compétences critiques et sens de la justice, actes du colloque de Cerisy (5-12 juin 2001), Paris, Economica, coll. « Études sociologiques », p. 261-274.

OGIEN Albert, 2014, «Pragmatismes et sociologies », Revue française de sociologie, $\mathrm{n}^{\circ} 3$, vol. 55 , p. 563-579. Disponible en ligne, https://www.cairn.info/revue-francaise-de-sociologie-2014-3page-563.htm [lien valide en juin 2018].

PEREC Georges, 1978, La Vie mode d'emploi, Paris, Hachette, coll « Club pour vous Hachette ». Pouloт Dominique, 2001, Patrimoine et Musées. L'institution de la culture, Paris, Hachette, coll. « Carré histoire ».

Poulot Dominique, 2006, Une histoire du patrimoine en Occident, XVIII ${ }^{e}-\mathrm{XXI}{ }^{e}$ siècle. Du monument aux valeurs, Paris, PUF, coll. « Le nœud gordien ».

RANCIÈrE Jacques, 1995, La Mésentente. Politique et philosophie, Paris, Galilée, coll. « La philosophie en effet ».

RUSKIN John, 2008 [1849], Les Sept lampes de l'architecture, Paris, Klincksieck, coll. « L'esprit et les formes ».

SANTos Boaventura de Sousa, 2011, «Épistémologies du Sud », Études rurales, n 187, « Le sens du rural aujourd'hui », p. 21-49. Disponible en ligne, https://journals.openedition.org/ etudesrurales/9351 [lien valide en juin 2018].

SANTOS Boaventura de Sousa, 2016, Épistémologies du Sud. Mouvements citoyens et polémiques sur la science, Paris, Desclée de Brouwer, coll. « Solidarité et société ».

SERVIGNE Pablo \& STEVEns Raphaël, 2015, Comment tout peut s'effondrer. Petit manuel de collapsologie à l'usage dans générations présentes, Paris, Éditions du Seuil, coll. « Anthropocène ».

SOURIAU Étienne, 2009 [1943, 1956], Les Différents Modes d'existence suivi de Du mode d'existence de l'œuvre à faire, Paris, PUF, coll. « MétaphysiqueS ».

STAVO-DEBAUGE Joan, 2012, « La sociologie dite "pragmatique” et la philosophie pragmatiste, une rencontre tardive », texte préparé pour le premier séminaire des Ateliers Villa-Vigoni, « Pourquoi le pragmatisme ? L'intérêt du pragmatisme pour les sciences humaines et sociales » (Villa Vigoni, Italie, 15-18 juillet 2012).

STENGERS Isabelle \& LATOUR Bruno, 2009, «Le Sphinx de l'œuvre », in SOURIAU Étienne, Les Différents Modes d'existence suivi de Du mode d'existence de l'œuvre à faire, Paris, PUF, coll. « MétaphysiqueS », p. 1-75.

STENGERS Isabelle, 2009, Au temps des catastrophes. Résister à la barbarie qui vient, Paris, La Découverte / Les empêcheurs de tourner en rond.

TORNATORE Jean-Louis, 2004a, « Beau comme un haut fourneau. Sur le traitement en monument des restes industriel », L'Homme, n 170, vol. 2, « Espèces d'objets », p. 79-116. 
TORNATORE Jean-Louis, 2004b, « La difficile politisation du patrimoine ethnologique », Terrain, $\mathrm{n}^{\circ}$ 42, « Homme / femme », p. 149-160. Disponible en ligne, https://journals.openedition.org/ terrain/1791 [lien valide en juin 2018].

TORNATORE Jean-Louis, 2006, « Les formes d'engagement dans l'activité patrimoniale. De quelques manières de s'accommoder au passé », in MEYER Vincent \& WALTER Jacques (dir.) Formes de l'engagement et espace public, Nancy, Presses universitaires de Nancy, coll. «Questions de communication », série « Actes », p. 515-538.

TORNATORE Jean-Louis, 2010a, « L'esprit de patrimoine », Terrain, $\mathrm{n}^{\circ}$ 55, « transmettre », p. 106-127. Disponible en ligne, https://journals.openedition.org/terrain/14084 [lien valide en juin 2018].

TORNATORE Jean-Louis, 2010b, « La reconnaissance pour mémoire. Un parcours dans l'espace de la mémoire de la "Lorraine sidérurgique" ", in TORNATORE Jean-Louis (dir.), L'Invention de la Lorraine industrielle. Quêtes de reconnaissance, politiques de la mémoire », Paris, Riveneuve éditions, coll. « Actes académiques », p. 161-191.

TORNATORE Jean-Louis, 2011, « Du patrimoine ethnologique au patrimoine culturel immatériel : suivre la voie politique de l'immatérialité culturelle », in воRтоцотто Chiara (dir.), Le patrimoine culturel immatériel. Enjeux d'une nouvelle catégorie, Paris, Éditions de la Maison des sciences de l'homme, p. 213-232.

TORNATORE Jean-Louis, 2014, «Words for expressing what we care about. The continuity and the exteriority of the heritage experience ", in BONDAZ Julien, GRAEZER BIDEAU Florence, ISNART Cyril \& LEBLON Anaïs (dir.), Les Vocabulaires locaux du «patrimoine ». Traductions, négociations et

transformations, Zürich, Lit Verlag, coll. « Freiburger sozialanthropologische Studien », p. 31-54.

TORNATORE Jean-Louis, 2017, « Questions de démocratie patrimoniale », in AUCLAIR Élizabeth, H ERZOG Anne \& POULOT Marie-Laure, De la participation à la co-construction des patrimoines urbains : l'invention du commun ?, Éditions Le Manuscrit, p. 15-45.

TROMPETTE Pascale \& VINCK Dominique, 2009, « Introduction », Revue d'anthropologie des connaissances, vol. 3, $\mathrm{n}^{\circ} 1$, « Retour sur la notion d'objet-frontière », p. 5-27. Disponible en ligne, https://www.cairn.info/revue-anthropologie-des-connaissances-2009-1-page-5.htm [lien valide en juin 2018].

voISENAT Claudie, 2016, « Le tournant patrimonial », in HOTTIN Christian, voISENAT Claudie (dir.), Le Tournant patrimonial. Mutations contemporaines des métiers du patrimoine, Paris, Éditions de la Maison des sciences de l'homme / ministère de la Culture, coll. «Ethnologie de la France », p. 17-42.

\section{NOTES}

1. Ce texte a été écrit durant l'été 2017. Il était initialement associé au texte de Noël Barbe, ce qui permet de comprendre l'usage ici ou là du «nous ». Il constituait également une réponse à la proposition d'écrire une histoire positiviste de la mission du patrimoine ethnologique, faite par Christian Hottin lors du colloque « Du moment du patrimoine ethnologique » (Dijon, décembre 2016) et qu'il prévoyait de développer dans ce numéro. Le débat n'a pas eu / n'aura cependant pas lieu.

2. https://pragmataaep.wordpress.com/ [lien valide en septembre 2018]. 
3. Le constat de cette diversité fait au moins l'unanimité. Comme l'écrit Albert Ogien (2014 : 564), « il n'existe pas de définition canonique du pragmatisme, ce qui autorise à l'invoquer, de façons également légitime, à l'appui des démarches les plus opposées en apparence ».

4. Sur la volonté de « réappropriation » de la critique par la sociologie pragmatique, voir Barthe et al. (2013 : 200 sq.). Dans ce même article, on trouvera une clarification sur la différence entre sociologie pragmatique et pragmatisme - que je ne prends pas en compte ici. Voir également Joan Stavo-Debauge (2012).

5. Montréal, juin 2016.

6. Laurajane Smith a été l'initiatrice, en 2012, de l'Association of Critical Heritage Studies et sa première présidente.

7. La question de la qualification hybride de ces forums, au sens où l'entendent Callon, Lascoumes \& Barthe (2001) reste ouverte.

8. Sur la position de croyant - que serait, par exemple, un conservateur du patrimoine - en contraste avec celle d'athée, i.e. de critique, voir l'article de Christoph Brumann (2014) qui plaide pour une tierce position agnostique.

9. J'ai organisé avec Noël Barbe deux manifestations: un séminaire «L'instauration du patrimoine ethnologie. Projet scientifique, catégorie d'actions publiques et instrument de gouvernementalité : retour sur une expérience française » (Paris, Lahic/ministère de la Culture, déc. 2010-sept. 2011), et un colloque «Du moment du patrimoine ethnologique » (Dijon, CGC/ IIAC, 7-8 décembre 2016) (publication en cours).

10. Voir la note 1.

11. On lui a préféré par la suite l'appellation d'« histoire méthodique » (Delacroix et al. 1999 : 97).

12. Selon Carbonell, l'histoire positiviste n'a de commun avec la philosophie comtienne que le nom, pis elle est en contradiction flagrante avec l'histoire soumise à des lois (de gradation, de continuité, de progrès...) qu'appellerait le positivisme. Estimant que "positiviste " relève du contresens, il juge davantage appropriée l'appellation d'« histoire positive ». " Prudence, amour du concret - du fait -, crainte de la non objectivité caractérisent l'esprit positif » (Carbonell 1978 : 183). On peut remarquer - ce qui d'ailleurs ne manque pas de sel - que le pragmatisme jamesien et le positivisme comtien entretiennent une certaine proximité. Pour James, ils se rejoignent «dans le mépris des solutions purement verbales, des fausses questions et des abstractions métaphysiques » (James $2007: 119)$. Cela ne va cependant guère plus loin.

13. Précisons que mon expérience de conseiller à l'ethnologie (en Direction régionale des affaires culturelles de Lorraine) s'est arrêtée en 2000, tandis que celle de Noël Barbe se poursuit. Il a été le plus loin dans l'expérimentation de cette articulation nouvelle entre pensée et action.

14. Pour quelques réflexions et pistes suggestives pour une histoire qui serait pragmatique et pragmatiste, voir Lepetit (1995), Offenstadt \& Van Damme (2009), Chateauraynaud \& Cohen (2016).

15. Voilà sans doute une invitation qui en elle-même n'est pas nouvelle. Il n'est cependant pas inutile de la réitérer tant le monde le monde politico-scientifique du patrimoine incline à essentialiser les catégories.

16. Il y a quelques années, j'ai essayé de dégager non pas une essence du patrimoine mais ce qui est visé à la fois avec et par-delà le mot, et que j'ai appelé alors «esprit de patrimoine » (Tornatore 2010). Le risque d'une telle appellation - même si de au lieu de du est censé l'en prévenir - est qu'elle accrédite l'idée classique qu'il y a une réalité spirituelle constituant un modèle ou un moule de la réalité objectale.

17. La notion d'instauration est empruntée à Étienne Souriau (2009) commenté par Isabelle Stengers et Bruno Latour (2009). Démarquée des idées de création comme de construction, elle met l'accent sur le fait qu'une œuvre, œuvre d'art ou tout autre être, « va vers l'existence » avec son instaurateur, au cours d'un trajet fait de liberté, d'efficacité et d'errabilité, " une existence qui à la fin éclatera de présence actuelle, intense, accomplie » (Souriau 2009 : 107). Un trajet et 
non un projet, car l'œuvre à faire se révèle dans l'être considéré comme accompli en son mode d'existence.

18. La question de l'attachement a été formulée par Bruno Latour (2000) - la lecture de ce texte m'a suggéré sa possible application au patrimoine (Tornatore 2007) - et développée par Antoine Hennion $(2010,2015)$ chez qui on trouve ce jeu sur le double sens du verbe tenir. La formule « ce à quoi nous tenons " a connu un certain succès : voir les publications concomitantes d'Émilie Hache (2011) et Alexandra Bidet, Louis Quéré et Jérôme Truc (2011) - « Ce à quoi nous tenons est aussi ce par quoi nous tenons» (ibid.: 33). Je l'ai reprise et appliquée au patrimoine dans une version sensiblement équivalente : «ce à quoi nous tenons et dont nous dépendons » (Tornatore 2014).

19. Les actions patrimoniales dont l'ethnologue en région a été à la fois l'acteur et le témoin.

20. Voir par exemple la justification par John Ruskin (2008 : 201) de l'obligation de conserver aux monuments leur intégrité dans le temps: «Nous n'avons pas le droit d'y toucher! Ils ne nous appartiennent pas. Ils appartiennent en partie à ceux qui les ont construits et en partie à toutes les générations d'hommes qui viendront après nous.» On peut citer également le "pragmatisme» de militants allemands pour la sauvegarde de hauts fourneaux qui élaborent le concept de « ruine contrôlée » afin d'assurer le maintien de machines promises à la casse. Ils fondent leur action de valuation sur l'argument suivant : nous ne savons pas ou nous n'avons pas les moyens de conserver ces machines selon les règles de l'art patrimonial, assurons simplement la possibilité de les transmettre aux générations futures, i.e. laissons leur la possibilité de faire avec, transmettons-leur un problème que nous ne savons pas régler (Tornatore 2004).

21. Voir infra l'apport de Boaventura de Sousa Santos (2016) sur ce point, et la note 44.

22. Selon John Dewey (2012b), l'intégrité globale de l'expérience - ou encore sa dimension inclusive - ne peut être véritablement reconnue que par la méthode empirique - «empirisme radical » (James 2005) ou « immédiat » (Dewey 2012a).

23. Sur la notion de savoir situé, voir Haraway (2009).

24. La paternité de la formule revient sans doute à Christian Jacquelin.

25. Voir les travaux de Chiara Bortolotto, en particulier Bortolotto (2015). Après plus de dix ans de mise en œuvre de la Convention de 2003, la fameuse injonction de participation s'est cependant révélé être un miroir aux alouettes.

26. En particulier sous l'impulsion de Daniel Fabre : voir Fabre (2016).

27. C'est ce que demande la Convention de 2003.

28. Comme l'écrit Christophe Charle (2011: 42), « le premier projet patrimonial [i.e. le moment 1830] s'affirme élitiste, centraliste, volontariste, artiste, même si, çà et là, il peut être relayé par des sociétés savantes ou des antiquaires plus éclairés ».

29. Tel est le projet de revisite générale de l'histoire du XIX ${ }^{\mathrm{e}}$ siècle prôné par Emmanuel Fureix et François Jarrige (2015). L’idée de la modernité comme « discordance des temps » est développée par Christophe Charle (2011).

30. La législation des monuments historiques mise en place au $\mathrm{XIX}^{\mathrm{e}}$ siècle réduit certes les prérogatives de la propriété privée au nom d'un intérêt national et met en concurrence deux formes d'appropriation. On sait combien l'ordre économique a su s'en accommoder.

31. «Le patrimoine enrôle les énergies citoyennes en faveur d'une culture de gouvernement : en montrant d'abord que le pouvoir actuel s'inscrit dans un long progrès, en ranimant ensuite une individualité assagie, voire énervée par les circonstances, à son profit »(Poulot 2006 : 148).

32. On peut prendre comme point de départ, la définition de l'arène publique qu'en donne Daniel Cefaï $(2016: 38)$ : «Une arène sociale dont les acteurs visent des biens publics, se réfèrent à l'intérêt public, définissent leurs problèmes comme publics et sentent, agissent et parlent en conséquence. » Elle diffère du marché (logique du profit), du champ (logique de domination) et de l'agora (logique de la délibération) qui ne sont que « des sous-espèces du modèle de l'arène 
sociale»(ibid.). La question reste cependant de savoir dans quelle mesure le profit ou la domination - et a fortiori la délibération - n'irriguent pas une arène publique.

33. Ainsi Mérimée évoque-t-il « le beau mouvement d'enthousiasme monumental » que suscite la « découverte » à Poitiers, en 1845, de la façade du Palais de justice, à la suite de la démolition de baraques qui en cachaient la vue (Mérimée 1998 : 149).

34. Les notions de "public» et de «situation problématique", ainsi que l'accent mis sur l'engagement de personnes dites ordinaire dans un travail d'«enquête " réfèrent au pragmatisme de John Dewey $(1967,2003)$. Pour un commentaire éclairant, voir Cefaï (2016).

35. On appréciera le basculement entre le point de départ de notre réflexion (agent de l'État) et cet aboutissement actuel. Est-ce la confirmation que l'État a changé ou bien une invitation à ne pas hypostasier l'État : permettant dans ses marges ou ses interstices sans se départir cependant d'une implacable ou inéluctable orientation générale?

36. Voir également les réserves émises par Ellen Hertz (2015) sur la notion de « bottom» (dans l'expression "bottom-up »), dans la situation de la prise suisse de la convention unesquienne de 2003.

37. "Anthropocène » désigne le problème mais reste vague ou ambigu quant aux solutions (que faire si toute l'humanité, indistinctement, est responsable de sa catastrophe ?), telle est la principale et radicale critique faite à ce terme. Parmi les désignations alternatives proposées, celle de « capitalocène » (Moore 2914) a l'avantage de coller davantage à la réalité de l'action des humains, et de suggérer que la solution au problème réside dans le rejet radical de sa cause : le capitalisme.

38. D'autant qu'ils sont saisis dans une logique capitaliste de consommation qui finit par être contre-productive pour les populations qui les habitent. Voir les oppositions de plus en plus violentes, durant l'été 2017, à la pression touristique.

39. Ainsi les militant.e.s et habitant.e.s des ZAD ne s'y reconnaissent absolument pas, ce que l'on peut tout à fait comprendre quand on a pu lire que l'élection du nouveau président de la République a résulté d'une mobilisation citoyenne orchestrée par le mouvement «La République en marche ». Pour une anthropologie de la citoyenneté, considérée dans sa portée capacitaire et non dans sa dimension statutaire, voir les travaux de Catherine Neveu (2005).

40. C'est en ce sens que l'expérience publique relève d'une écologie humaine (Cefaï 2016).

41. Voir l'article « Faire de la vie sociale un art » (Multitudes 2017 : 38-41).

42. La relation de connivence et l'expression d'une contribution militante peuvent se manifester en investissant les moyens et procédures du monde académique - bien que l'interrogation de leur dépassement subsiste - : voir le séminaire tenu à l'EHESS depuis 2016 et animé avec Noël Barbe «Expériences de l'anticapitalisme », et l'atelier de recherche du Centre Georges-Chevrier, animé avec François Jarrige, «Penser la/les transition-s ». On remarquera en outre que si le scénario proposé s'inscrit dans une écologie de l'expérience publique, il se démarque sensiblement, par cette orientation politique affichée, du modèle des arènes publiques expérimenté dans les ÉtatsUnis du début du XX $\mathrm{XX}^{\mathrm{e}}$ siècle, auquel ont collaboré les sociologues de Chicago, et exposé en guise d'exemple paradigmatique par Daniel Cefaï (2016).

43. Autour des travaux de Philippe Descola, Eduardo Viveiros de Castro, Eduardo Kohn, Anna Tsing... Voir le dossier de la revue Tsantsa dirigé par Frédéric Keck, Ursula Regehr et Saskia Walentowitz (2015). Voir également l'ouvrage récemment traduit d'Arturo Escobar (2018).

44. Soleil vert (Soylent Green) est un film de Richard Fleisher, sorti en 1973. Dans un monde surpeuplé, bétonné et devenu stérile, où la matière humaine est la seule ressource exploitable pour la survie de l'humanité, l'euthanasie est désormais un service proposé - un service social -, auquel se résout un des protagonistes du film lorsqu'il prend conscience que le "Soylent green » que la population consomme n'est pas fabriqué à partir du plancton mais à partir des cadavres humains. Il meurt en visionnant des images montrant la beauté naturelle et sauvage du monde 
perdu sur une musique appropriée, en l'occurrence ce sirop beethovenien qu'est La Symphonie pastorale.

45. En particulier celle qui se demande si « l'ontologie n'est pas juste un autre mot pour culture » (Carrithers, Candea, Sykes, Holbraad \& Venkatesan 2010).

46. Voir les travaux de Diego Landivar et Émilie Ramillien (2015) sur les nouvelles constitutions andines en Bolivie et en Équateur.

47. Laquelle est une « raison métonymique »: « une sorte de raison qui prétend être la seule forme de rationalité » (Santos $2016: 242$ ).

48. Dans la pensée hégémonique, le présent est un instantané vide ne prenant sens que par rapport au passé ou au futur, ce qui explique la « dénonciation » du présentisme en tant qu'éloge d'un présent effarant. La pensée contre-hégémonique inverse la relation en considérant que le passé, et surtout le futur, prennent sens dans un présent qui leur assure une pleine présence. Le récent ouvrage de Jérôme Baschet (2018) offre une réflexion renouvelée sur les régimes d'historicité, qui permet de penser la temporalité contre-hégémonique.

49. Thèse de Marc Guillaume (1980) inspirée par Michel de Certeau.

50. Voir également la notion de «fratricides rassurants » avancée par Bénédict Anderson (1996).

51. Ces fonctions patrimoniales sont particulièrement lisibles dans le cas des patrimonialisations des restes de la seconde industrialisation (Tornatore 2010).

52. Situation dont s'est emparé George Perec (1978: 144-150) dans La Vie, mode d'emploi, en narrant la tragique histoire de l'ethnologue Marcel Appenzzel.

53. Mon usage de ce mot peut être considéré comme abusif au regard d'une définition anthropologique et canonique de la transe. Je retiens de son étymologie l'idée d'un passage (trans ire, aller au-delà) signifié par un changement d'état, lequel peut être congruent à une hypersensibilité - quasi-photographique - constitutive du fait d'« être sur le terrain ».

54. L'idée d'un art de transe découlerait de son caractère spectaculaire et de sa proximité avec le théâtre. Voir le dossier de la revue Gradhiva consacré au thème: "possession, théâtre et globalisation » (Dianteill \& Hell 2008).

55. La déprise, au sens de se déprendre des gestes et attitudes communément signifiants afin de pouvoir donner libre cours au travail du corps, est le principe du travail théâtral dans la conception formulée dans les années 1960-1970 par le théoricien du «théâtre pauvre ", Jerzy Grotowski (1971), et qu'il désigne comme "voie négative » - par rapport à la voie positive qui consiste à apprendre des attitudes, des gestes et des trucs signifiants dans la pratique théâtrale d'une culture donnée. Je vois le pragmatisme comme une sorte de voie négative permettant d'assurer une compréhension non pré-formatée, non instruite par des catégories préconçues, du travail des acteurs sociaux.

56. Sur la question de la confiscation des mots et le refus de les laisser "à l'ennemi », voir l'ouvrage récent de Marie-José Mondzain (2017). Son point de départ, la question du radicalisme, est cependant différent du mien, puisque le mot « radical » est frappé d'ignominie du fait de la référence récente au terrorisme religieux alors que « patrimoine » est historiquement investi.

\section{RÉSUMÉS}

À partir de plusieurs constats, le débordement des institutions patrimoniales, l'insistance de formes citoyennes ou plébéiennes des revendications d'attachements, la prégnance de la figure 
du vivant, la raréfaction du futur devant la perspective anthropocénique, ce texte voudrait jeter les bases d'un scénario contre-hégémonique du patrimoine : détaché des institutions d'État, loin du marché et de sa logique néo-libérale, engagé dans des expériences de problèmes publics, sensible à la pluralité ontologies et des modes de connaissance...

Some preliminary observations: too much is now asked of heritage institutions; new citizens' and plebeian forms are emerging of asserting attachments; life-science figures are becoming pervasive; the future is disappearing as a result of anthropocenic harmfulness... Based on such observations, the present text would like to set out the foundations for a counter-hegemonic heritage scenario, freed from state institutions, independent of the market and its neo-liberal logics, engaged with the public experimentation of problems and sensitive to the plurality of ontologies and modes of knowledge.

INDEX

Keywords : heritage, ethnological heritage, pragmatism, plebeian, citizen, experiment, public problems, ontology, epistemology, involvement, trance

Mots-clés : patrimoine, patrimoine ethnologique, pragmatisme, plébéien, citoyen, expérience, problèmes public, ontologie, épistémologie, engagement, transe

\section{AUTEUR}

\section{JEAN-LOUIS TORNATORE}

Professeur en anthropologie, université de Bourgogne, membre du Centre Georges-Chevrier (UMR CNRS 7366) 